Y. Ando

Nagoya Math. J.

Vol. 164 (2001), 147-184

\title{
ON HOLOMORPHIC MAPS WITH ONLY FOLD SINGULARITIES
}

\author{
YOSHIFUMI ANDO ${ }^{1}$
}

\section{Dedicated to Professor Takuo Fukuda on his sixtieth birthday}

\begin{abstract}
Let $f: N \rightarrow P$ be a holomorphic map between $n$-dimensional complex manifolds which has only fold singularities. Such a map is called a holomorphic fold map. In the complex 2-jet space $J^{2}(n, n ; \mathbf{C})$, let $\Omega^{10}$ denote the space consisting of all 2 -jets of regular map germs and fold map germs. In this paper we prove that $\Omega^{10}$ is homotopy equivalent to $\mathrm{SU}(n+1)$. By using this result we prove that if the tangent bundles $T N$ and $T P$ are equipped with $\mathrm{SU}(n)$-structures in addition, then a holomorphic fold map $f$ canonically determines the homotopy class of an $\mathrm{SU}(n+1)$-bundle map of $T N \oplus \theta_{N}$ to $T P \oplus \theta_{P}$, where $\theta_{N}$ and $\theta_{P}$ are the trivial line bundles.
\end{abstract}

\section{Introduction}

Let $N$ and $P$ be complex manifolds of dimension $n$. We shall say that a holomorphic map germ of $(N, x)$ to $(P, y)$ has a fold singularity at $x$ if it is written as $\left(z_{1}, \ldots, z_{n-1}, z_{n}\right) \mapsto\left(z_{1}, \ldots, z_{n-1}, z_{n}^{2}\right)$ under suitable local coordinate systems near $x$ and $y$. Such a germ will be called a fold map germ. A holomorphic map $f: N \rightarrow P$ will be called a holomorphic fold map if $f$ has only fold singularities.

Let $J^{k}(n, n ; \mathbf{C})\left(J^{k}(n, n)\right.$ for short) denote the $k$-jet space of all $k$-jets of holomorphic map germs $\left(\mathbf{C}^{n}, \mathbf{0}\right) \rightarrow\left(\mathbf{C}^{n}, \mathbf{0}\right)$. We consider the subspace $\Omega^{1}$ of $J^{1}(n, n)$ consisting of all 1 -jets whose kernel rank is either 0 or 1 , and the subspace $\Omega^{10}$ of $J^{2}(n, n)$ consisting of all 2-jets of regular germs and fold map germs. The purpose of this paper is to determine their homotopy types. Let $J^{2}(N, P ; \mathbf{C})\left(J^{2}(N, P)\right.$ for short $)$ denote the complex 2-jet space, which is the total space of a fibre bundle over $N \times P$ and $\Omega^{10}(N, P ; \mathbf{C})\left(\Omega^{10}(N, P)\right.$

Received December 1, 1998.

Revised February 22, 2000.

2000 Mathematics Subject Classification: Primary 58K15; Secondary 58A20, 58C10.

${ }^{1}$ This research was partially supported by Grant-in-Aid for Scientific Research (No.11640081), Ministry of Education, Science and Culture, Japan. 
for short) denote its subbundle associated with $\Omega^{10}$. The the 2 -jet extension $j^{2} f$ of a holomorphic fold map $f: N \rightarrow P$ is a section of $\Omega^{10}(N, P)$ over $N$. The homotopy type of $\Omega^{10}$ will be important in the study of the space consisting of all holomorphic fold maps. This paper is partially the complex version of [A1] and [A2], although the arguments are quite different and more complicated except for Sections 1 and 2.

Let $S^{2 k-1}, D_{r}^{2 k}$ and $\mathbf{C P}^{k-1}$ denote the unit sphere of dimension $2 k-1$ in $\mathbf{C}^{k}$, the disk of radius $r$ and of dimension $2 k$ in $\mathbf{C}^{k}$, and the complex projective space of dimension $k-1$ respectively. Let $\mathrm{U}(k)$ and $\mathrm{SU}(k)$ denote the unitary group and the special unitary group of degree $k$ respectively. Now we explain the homotopy types of $\Omega^{1}$ and $\Omega^{10}$. Let $I_{a}(a \in \mathbf{R})$ be the diagonal $n \times n$-matrix ( $n$-matrix for short) with diagonal components $\left(1, \ldots, 1, e^{\sqrt{-1} a}\right)$. Let $\mathbf{v}$ be a point of $\mathbf{C P}^{n-1}$ represented by a vector $\mathbf{s}=$ ${ }^{t}\left(s_{1}, s_{2}, \ldots, s_{n}\right)$ of $S^{2 n-1}$. Then we define the $n$-matrix $G\left(\mathbf{v}, e^{\sqrt{-1} \theta}\right)$ by

$$
G\left(\mathbf{v}, e^{\sqrt{-1} \theta}\right)=I_{\theta}\left(E_{n}+\left(e^{\sqrt{-1} \theta}-1\right)\left(s_{i} \bar{s}_{j}\right)\right)
$$

where $E_{n}$ is the unit matrix of rank $n$ and $\left(s_{i} \bar{s}_{j}\right)$ is the $n$-matrix with $(i, j)$ component given by $s_{i} \bar{s}_{j}$. It will be shown that $G\left(\mathbf{v}, e^{\sqrt{-1} \theta}\right)$ lies in $\operatorname{SU}(n)$ (see (3.3)). Let $\mathrm{OC}\left(\mathbf{C} \mathbf{P}^{n-1}\right)$ denote the open cone over $\mathbf{C} \mathbf{P}^{n-1}$, that is, the quotient space $\mathbf{C P}^{n-1} \times[0,1) / \mathbf{C P}^{n-1} \times 0$. Then we define the homeomorphism

$$
g: \mathbf{C P}^{n-1} \times \operatorname{Int}\left(D_{1 / 2}^{2} \backslash\{\mathbf{0}\}\right) \times \mathrm{SU}(n) \longrightarrow \mathbf{C P}^{n-1} \times(\sqrt{3} / 2,1) \times S^{1} \times \mathrm{SU}(n)
$$

by $g\left(\mathbf{v}, b e^{\sqrt{-1} \theta}, U\right)=\left(\mathbf{v},\left(1-b^{2}\right)^{1 / 2}, e^{\sqrt{-1} \theta}, G\left(\mathbf{v}, e^{\sqrt{-1} \theta}\right) U\right)$. We make the new space $\mathbf{C P}^{n-1} \times \operatorname{Int} D_{1 / 2}^{2} \times \mathrm{SU}(n) \cup_{g} \mathrm{OC}\left(\mathbf{C P}^{n-1}\right) \times S^{1} \times \mathrm{SU}(n)$ by pasting the two subspaces by $g$.

We consider the two actions of $\mathrm{SU}(n) \times \mathrm{SU}(n)$ : one on $J^{2}(n, n)$ through the source and target spaces $\left(\mathbf{C}^{n}, \mathbf{0}\right)$, and the other on $\mathrm{SU}(n+1)$ through $\mathrm{SU}(n) \times(1)$ from the right and left hand sides. The main theorem of the present paper is the following.

THEOREM 1. (1) There exists a topological embedding of $\mathbf{C P}^{n-1} \times$ Int $D_{1 / 2}^{2} \times \mathrm{SU}(n) \cup_{g} \mathrm{OC}\left(\mathbf{C P}^{n-1}\right) \times S^{1} \times \mathrm{SU}(n)$ into $\Omega^{1}$ whose image is a deformation retract of $\Omega^{1}(n \geq 2)$.

(2) There exists an equivariant topological embedding $i_{n}: \mathrm{SU}(n+1) \rightarrow$ $\Omega^{10}$ with respect to the actions of $\mathrm{SU}(n) \times \mathrm{SU}(n)$ whose image is a deformation retract of $\Omega^{10}(n \geq 1)$. 
An $n$-dimensional complex vector bundle with structure group $\mathrm{SU}(n)$ will be called an $\mathrm{SU}(n)$-vector bundle. Let $M$ be a complex manifold of dimension $n$. In this paper, an $\mathrm{SU}(n)$-structure of $T M$ refers to a reduction $\left(E^{\prime}, \varphi\right)$ of the structure group $\mathrm{GL}(n, \mathbf{C})$ of the tangent bundle $T M$ to $\mathrm{SU}(n)$, where $E^{\prime}$ is an $\mathrm{SU}(n)$-vector bundle over $M$ and $\varphi: T M \rightarrow E^{\prime}$ is a bundle map. Then $\left(E^{\prime}, \varphi\right)$ induces a homotopy class of a classifying map of $E^{\prime}$, $M \rightarrow B_{\mathrm{SU}(n)}$. It is well known that $T M$ has an $\mathrm{SU}(n)$-structure if and only if the first Chern class of $M$ vanishes.

Let $L^{2}(n)$ be the group of all 2-jets of biholomorphic map germs $\left(\mathbf{C}^{n}, \mathbf{0}\right)$ $\rightarrow\left(\mathbf{C}^{n}, \mathbf{0}\right)$. The structure group of the fibre bundle $\pi_{N} \times \pi_{P}: J^{2}(N, P) \rightarrow$ $N \times P$ with fibre $J^{2}(n, n)$ is $L^{2}(n) \times L^{2}(n)$. Since $\mathrm{GL}(n, \mathbf{C})$ is naturally a subgroup of $L^{2}(n)$ and the quotient space $L^{2}(n) / \mathrm{GL}(n ; \mathbf{C})$ is contractible, the structure group $L^{2}(n) \times L^{2}(n)$ of the fibre bundle $\pi_{N} \times \pi_{P}: J^{2}(N, P) \rightarrow$ $N \times P$ is reduced to $\operatorname{GL}(n ; \mathbf{C}) \times \operatorname{GL}(n ; \mathbf{C})$. If $T N$ and $T P$ have $\mathrm{SU}(n)$ structures $\left(E, \varphi_{N}\right)$ and $\left(F, \varphi_{P}\right)$ respectively, then the structure group of $J^{2}(N, P)$ is, furthermore, reduced from $\mathrm{GL}(n ; \mathbf{C}) \times \mathrm{GL}(n ; \mathbf{C})$ to $\mathrm{SU}(n) \times$ $\mathrm{SU}(n)$. Moreover, we have the subbundle $\mathrm{SU}\left(E \oplus \theta_{N}, F \oplus \theta_{P}\right)$ of $\operatorname{Hom}(E \oplus$ $\left.\theta_{N}, F \oplus \theta_{P}\right)$ associated with $\mathrm{SU}(n+1)$, where $\theta_{N}$ and $\theta_{P}$ are the trivial complex line bundles over $N$ and $P$ respectively. We will prove in Section 7 that there exists a fibre map $i(N, P): \mathrm{SU}\left(E \oplus \theta_{N}, F \oplus \theta_{P}\right) \rightarrow \Omega^{10}(N, P)$ associated with the equivariant embedding $i_{n}: \mathrm{SU}(n+1) \rightarrow \Omega^{10}$ in Theorem 1 (2). The $\mathrm{SU}(n)$-vector bundles $E$ and $F$ not only have hermitian metrics, but also enable us to consider the determinant on each fibre of a bundle map of $E$ to $F$. A bundle map of $E$ to $F$ will be called an SU(n)-bundle map if it preserves norms and the determinant on each fibre is equal to 1 . The following theorem will be proved in Section 7 .

THEOREM 2. Let $N$ and $P$ be complex manifolds of dimension $n$ with $\mathrm{SU}(n)$-structures $\left(E, \varphi_{N}\right)$ and $\left(F, \varphi_{P}\right)$ respectively. Then we have the following.

(1) The map $i(N, P): \mathrm{SU}\left(E \oplus \theta_{N}, F \oplus \theta_{P}\right) \rightarrow \Omega^{10}(N, P)$ is a fibre homotopy equivalence.

(2) If there exists a holomorphic fold map $f$ of $N$ into $P$, then $j^{2} f$ determines the homotopy class of an $\mathrm{SU}(n+1)$-bundle map of $E \oplus \theta_{N}$ to $F \oplus \theta_{P}$ covering $f$ through $i(N, P)$.

The set of all continuous sections of $\mathrm{SU}\left(E \oplus \theta_{N}, F \oplus \theta_{P}\right)$ over $N$ corresponds bijectively to that of all $\mathrm{SU}(n+1)$-bundle maps of $E \oplus \theta_{N}$ to $F \oplus \theta_{P}$. 
For a holomorphic fold map $f$, the section $j^{2} f: N \rightarrow \Omega^{10}(N, P)$ determines the homotopy class of the section $i(N, P)^{-1} \circ j^{2} f$ of $\operatorname{SU}\left(E \oplus \theta_{N}, F \oplus \theta_{P}\right)$, where $i(N, P)^{-1}$ is the homotopy inverse of $i(N, P)$. This gives the homotopy class of an $\mathrm{SU}(n+1)$-bundle map $\tilde{f}: E \oplus \theta_{N} \rightarrow F \oplus \theta_{P}$ covering $f$ in Theorem 2 (2). Since $\widetilde{f}$ is reduced to an $\mathrm{SU}(n)$-bundle map of $E$ to $F$ by the obstruction theory, we have the following corollary.

Corollary 3. Let $N$ and $P$ be complex manifolds of dimension $n$ whose first Chern classes vanish. If there is a holomorphic fold map $f$ : $N \rightarrow P$, then there exists a bundle map of TN to TP covering $f$.

The assertion in the $C^{\infty}$-category corresponding to Theorem 2 is described in [A2, Corollary 2] and Corollary 3 can be compared with the results [E, 3.8, 3.9 and 3.10 Theorem] and [Sa, Lemma 3.1] in the $C^{\infty}$ category.

In Section 2 we will prepare lemmas in linear algebra. Let $\Sigma^{1}$ denote the subspace of $J^{1}(n, n)$ consisting of all 1-jets with kernel rank 1 . We will prove in Section 3 that $\Sigma^{1}$ is homotopy equivalent to $\mathbf{C P}^{n-1} \times \mathrm{SU}(n)$ (Theorems 3.1 and 3.7). It is known that the normal bundle of $\Sigma^{1}$ in $\Omega^{1}$ is the trivial complex line bundle $\operatorname{Hom}(\mathbf{K}, \mathbf{Q})$, where $\mathbf{K}$ is the kernel bundle and $\mathbf{Q}$ is the cokernel bundle of the first derivative over $\Sigma^{1}$. Therefore the tubular neighbourhood of $\Sigma^{1}$ is homotopy equivalent to $\mathbf{C P}^{n-1} \times D_{1 / 2}^{2} \times \mathrm{SU}(n)$. We will study how $\partial\left(\mathbf{C P}^{n-1} \times D_{1 / 2}^{2} \times \mathrm{SU}(n)\right)$ is pasted to $\mathrm{U}(n) \cong S^{1} \times \mathrm{SU}(n)$

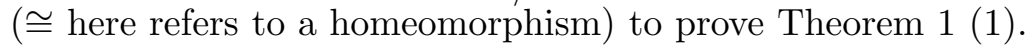

Let $\Sigma^{10}$ denote the subspace of $J^{2}(n, n)$ consisting of all 2-jets of fold map germs. In Section 5 we will see that the fibre bundle $\Sigma^{10}$ over $\Sigma^{1}$ is homotopy equivalent to the canonical $S^{1}$-bundle $S^{2 n-1} \times \mathrm{SU}(n)$ over $\mathbf{C P}^{n-1} \times \mathrm{SU}(n)$ and hence the tubular neighbourhood of $\Sigma^{10}$ in $\Omega^{10}$ is homotopy equivalent to $S^{2 n-1} \times D_{1 / 2}^{2} \times \mathrm{SU}(n)$. The tubular neighbourhood of $\left.U(n) \cong S^{1} \times \mathrm{SU}(n)\right)$ in $\Omega^{10}$ is homotopy equivalent to $D_{1}^{2 n} \times S^{1} \times \mathrm{SU}(n)$. Then we will see that the pasting map of $\partial\left(S^{2 n-1} \times D_{1 / 2}^{2} \times \mathrm{SU}(n)\right)$ to $\partial\left(D_{1}^{2 n} \times S^{1} \times \mathrm{SU}(n)\right)$ is induced from $g$ by considering the $S^{1}$-bundle above and that the pasted space becomes the total space of a fibre bundle over $S^{2 n+1}$ with fibre SU $(n)$. We will prove in Section 5 that there exists a bundle map from this space to $\mathrm{SU}(n+1)$ by constructing in Section 4 a special bundle structure of the fibre bundle $\mathrm{SU}(n+1)$ over $\mathrm{SU}(n+1) / \mathrm{SU}(n) \times$ $\mathrm{SU}(1) \cong S^{2 n+1}$. 
Next we will specify the embedding of $\mathrm{SU}(n+1)$ into $\Omega^{10}$ of Theorem $1(2)$ in Section 5 and prove in Section 6 that it is equivariant with respect to the actions of $\mathrm{SU}(n) \times \mathrm{SU}(n)$. In Section 7 we will prove Theorem 2 and give certain examples of holomorphic fold maps.

\section{$\S 1$. Notations}

Let $\mathbf{C}^{n}$ denote the $n$-dimensional complex number space consisting of all column vectors of $n$ complex numbers. Let $\left\{\mathbf{e}_{1}, \ldots, \mathbf{e}_{n}\right\}$ denote the canonical basis of $\mathbf{C}^{n}$ with $\mathbf{e}_{i}={ }^{t}\left(0, \ldots, 0,{ }_{1}^{i}, 0, \ldots, 0\right)$. The hermitian inner product of vectors $\mathbf{v}, \mathbf{w}$ is denoted by $(\mathbf{v}, \mathbf{w})$ and the norm of $\mathbf{v}$ is denoted by $\|\mathbf{v}\|$. In this paper a linear map $\mathbf{C}^{n} \rightarrow \mathbf{C}^{n}$ or a quadratic form on $\mathbf{C}^{n}$ is identified with an $n$-matrix or an $n$-symmetric matrix respectively.

The details and further results of this section can be found in [Bo] and [L] although we work in the complex category. The space of all homomorphisms of a vector space $V$ into a vector space $W$ over $\mathbf{C}$ will be denoted by $\operatorname{Hom}(V, W)$. The basis $\left\{\mathbf{e}_{1}, \ldots, \mathbf{e}_{n}\right\}$ induces the identifications of $J^{1}(n, n)$ with $\operatorname{Hom}\left(\mathbf{C}^{n}, \mathbf{C}^{n}\right)$ and of $J^{2}(n, n)$ with $\operatorname{Hom}\left(\mathbf{C}^{n}, \mathbf{C}^{n}\right) \oplus \operatorname{Hom}\left(\mathbf{C}^{n} \bigcirc \mathbf{C}^{n}, \mathbf{C}^{n}\right)$, where $\mathbf{C}^{n} \bigcirc \mathbf{C}^{n}$ is the 2-fold symmetric product of $\mathbf{C}^{n}$. Let $\Sigma^{i}$ denote the subspace of $J^{1}(n, n)$ consisting of all homomorphisms $\alpha: \mathbf{C}^{n} \rightarrow \mathbf{C}^{n}$ with kernel rank $i(0 \leq i \leq n)$. We usually denote an element of $J^{2}(n, n)$ as $(\alpha, \beta)$ for $\alpha: \mathbf{C}^{n} \rightarrow \mathbf{C}^{n}$ and $\beta: \mathbf{C}^{n} \bigcirc \mathbf{C}^{n} \rightarrow \mathbf{C}^{n}$. Consider the composition of the restriction $\beta \mid \operatorname{Ker}(\alpha) \bigcirc \operatorname{Ker}(\alpha)$ and the natural projection of $\mathbf{C}^{n}$ onto $\operatorname{Cok}(\alpha)$. It induces a new homomorphism of $\operatorname{Ker}(\alpha)$ into $\operatorname{Hom}(\operatorname{Ker}(\alpha), \operatorname{Cok}(\alpha))$ denoted by $\widetilde{\beta}$. Let $\Sigma^{i j}$ be the subspace consisting of all elements $(\alpha, \beta)$ such that $\alpha$ and $\widetilde{\beta}$ are of kernel ranks $i$ and $j$ respectively. The notation $\Sigma^{i}$ is often used for $\Sigma^{i} \times \operatorname{Hom}\left(\mathbf{C}^{n} \bigcirc \mathbf{C}^{n}, \mathbf{C}^{n}\right)$ if there is no confusion.

The space $\Omega^{1}$ denotes the union $\Sigma^{0} \cup \Sigma^{1}$ in $J^{1}(n, n)$ and $\Omega^{10}$ denotes the union $\Sigma^{0} \cup \Sigma^{10}$ in $J^{2}(n, n)$. Both spaces are open subsets. We say that a 2 -jet of $\Sigma^{10}$ or its singularity at the origin is of fold type.

In this paper maps are basically continuous, but may be holomorphic or $C^{\infty}$-differentiable if so stated.

\section{$\S 2$. Lemmas}

In this section we will discuss several results proved by elementary arguments in linear algebra in the complex category. The diagonal matrix with diagonal components $\mathbf{a}=\left(a_{1}, \ldots, a_{n}\right)$ will be denoted by $\Delta(\mathbf{a})$. In 
particular, $\Delta\left(1, \ldots, 1, e^{\sqrt{-1} a}\right)$ of rank $n$ is written as $I_{a}$. For an $n$-matrix $A,{ }^{t} \bar{A}$ is denoted by $A^{*}$.

Lemma 2.1. Let $A$ be an $n$-matrix. Then $A$ is decomposed as $S \Delta(\mathbf{d}) T$, where $S$ and $T$ are unitary matrices and $d_{1}, \ldots, d_{n}$ are nonnegative real numbers such that (1) $d_{1}^{2}, \ldots, d_{n}^{2}$ are the eigen-values of $A^{*} A$ and $(2) d_{1} \geq$ $d_{2} \geq \cdots \geq d_{n} \geq 0$.

Proof. The hermitian and nonnegative definite matrix $A^{*} A$ is diagonalized by a unitary matrix $U$ as

$$
U^{*}\left(A^{*} A\right) U=\Delta\left(d_{1}^{2}, \ldots, d_{n}^{2}\right)
$$

Set $U^{*} A U=\left(\mathbf{a}_{1}, \ldots, \mathbf{a}_{n}\right)$. Then $\left(\mathbf{a}_{i}, \mathbf{a}_{j}\right)=0$ for $i \neq j$ and $\left(\mathbf{a}_{i}, \mathbf{a}_{i}\right)=d_{i}^{2}$. When $\mathbf{a}_{i} \neq \mathbf{0}$, set $\mathbf{f}_{i}=\mathbf{a}_{i} /\left\|\mathbf{a}_{i}\right\|$. Then we can find an orthonormal basis $\mathbf{f}_{1}, \ldots, \mathbf{f}_{n}$ by choosing $\mathbf{f}_{j}$ for $j$ with $\mathbf{a}_{j}=\mathbf{0}$ appropriately. It follows that

$$
U^{*} A U=\left(\mathbf{f}_{1}, \ldots, \mathbf{f}_{n}\right) \Delta\left(\left\|\mathbf{a}_{1}\right\|, \ldots,\left\|\mathbf{a}_{n}\right\|\right)
$$

This proves (1).

We can prove that in the decomposition of $A$ two values $d_{i}$ and $d_{j}$ are exchanged by using the matrix $P_{i j}=\left(p_{i j}\right)$ such that $p_{k k}=1$ when $k$ is equal to neither $i$ nor $j$ and that $p_{i j}=p_{j i}=1$ and $p_{s t}=0$ otherwise. This follows from $A=S P_{i j} P_{i j} \Delta(\mathbf{d}) P_{i j} P_{i j} T$ and $P_{i j} \Delta(\mathbf{d}) P_{i j}=\Delta\left(d_{1}, \ldots, d_{j}, \ldots d_{i}, \ldots, d_{n}\right)$.

If $d_{1} \geq d_{2} \geq \cdots \geq d_{n} \geq 0$ holds, then we say in this paper that the diagonal components $\mathbf{d}=\left(d_{1}, \ldots, d_{n}\right)$ is decreasing. Let $A_{j}(j=1, \ldots, s)$ be square $i_{j}$-matrices. The new matrix

$$
\left(\begin{array}{cccc}
A_{1} & & & \\
& A_{2} & & 0 \\
& & \ddots & \\
& 0 & & A_{s}
\end{array}\right)
$$

will be denoted by $A_{1} \dot{+} \cdots \dot{+} A_{s}$. Let $E_{j}$ denote the unit matrix of rank $j$.

The following lemma is a key tool of this paper.

LEMMA 2.2. Let $\mathbf{v}$ and $\mathbf{w}$ be decreasing diagonal components. Suppose that $S \Delta(\mathbf{v}) T=\Delta(\mathbf{w})$ for $S$ and $T$ of $\mathrm{U}(n)$. Then 
(1) $\mathbf{v}=\mathbf{w}$. Hence $\Delta(\mathbf{v})(=\Delta(\mathbf{w}))$ is written as

$$
a_{1} E_{i_{1}} \dot{+} a_{2} E_{i_{2}} \dot{+} \cdots \dot{+} a_{s} E_{i_{s}}
$$

where $a_{1}, \ldots, a_{s}$ are all distinct and $n=i_{1}+\cdots+i_{s}$.

(2) $S$ and $T$ are also matrices of the forms

$$
S=S_{1} \dot{+} \cdots \dot{+} S_{s} \text { and } T=T_{1} \dot{+} \cdots \dot{+} T_{s}
$$

respectively, where both $S_{j}$ and $T_{j}$ are of ranks $i_{j}(j=1, \ldots, s)$.

(3) If $a_{j}$ is not zero, then $S_{j} T_{j}=E_{i_{j}}$.

Proof. We shall prove the lemma by comparing the components of $S \Delta(\mathbf{v})$ and $\Delta(\mathbf{w}) T^{*}$. Set $S=\left(s_{i j}\right)$ and $T^{*}=\left(t_{i j}\right)$. Then we have

$$
\left(\begin{array}{ccc}
v_{1} s_{11} & \ldots & v_{n} s_{1 n} \\
\vdots & & \vdots \\
v_{1} s_{n 1} & \ldots & v_{n} s_{n n}
\end{array}\right)=\left(\begin{array}{ccc}
w_{1} t_{11} & \ldots & w_{n} t_{1 n} \\
\vdots & & \vdots \\
w_{1} t_{n 1} & \ldots & w_{n} t_{n n}
\end{array}\right)
$$

By comparing the components of $p$-th rows and $q$-th columns of the matrices above, we obtain the following inequalities.

$$
\begin{aligned}
\left|v_{1}\right|^{2} & \geq\left|v_{1} s_{p 1}\right|^{2}+\left|v_{2} s_{p 2}\right|^{2}+\cdots+\left|v_{n} s_{p n}\right|^{2} \\
& =\left|w_{p} t_{p 1}\right|^{2}+\left|w_{p} t_{p 2}\right|^{2}+\cdots+\left|w_{p} t_{p n}\right|^{2} \\
& =\left|w_{p}\right|^{2} \\
\left|w_{1}\right|^{2} & \geq\left|w_{1} t_{1 q}\right|^{2}+\left|w_{2} t_{2 q}\right|^{2}+\cdots+\left|w_{n} t_{n q}\right|^{2} \\
& =\left|v_{q} s_{1 q}\right|^{2}+\left|v_{q} s_{2 q}\right|^{2}+\cdots+\left|v_{q} s_{n q}\right|^{2} \\
& =\left|v_{q}\right|^{2} .
\end{aligned}
$$

Setting $p=q=1$, we have $v_{1}=w_{1}$.

Now we prove the lemma by induction on $n$. Assume that the assertion is true for dimensions less than $n$. If $v_{n}=0$ or $w_{n}=0$, then the number of $i$ 's with $v_{i}=0$ coincides with that of $j$ 's with $w_{j}=0$. Let $i_{s}$ denote this number. By the unitarity of $S$ and $T$ it follows that $s_{p q}=t_{p q}=0$ when only one of $p$ and $q$ is smaller than $n-i_{s}+1$ and the other is not. So let $a_{s}=0, S_{s}$ and $T_{s}$ 
denote $i_{s}$-matrices $\left(s_{p q}\right)$ and $\left(t_{p q}\right)$, where $n-i_{s}+1 \leq p, q \leq n$, respectively. Therefore the assertion for $n$ follows from the induction hypothesis.

Next assume that $v_{i}$ and $w_{j}$ are not zero for all $i$ and $j$. Suppose that

$$
v_{1}=v_{2}=\cdots=v_{i}>v_{i+1} \quad \text { and } \quad w_{1}=w_{2}=\cdots=w_{i}>w_{j+1}
$$

Then we can prove that $i=j$ and $s_{p q}=t_{p q}=0$ when only one of $p$ and $q$ is smaller than $i+1$ and the other is not. In fact, if $p \leq j$, then (2.3.1) implies $\left|v_{1}\right|^{2} \geq\left|w_{p}\right|^{2}=\left|w_{1}\right|^{2}=\left|v_{1}\right|^{2}$ and so

$$
\begin{aligned}
\left|v_{1}\right|^{2} & =\left|v_{1} s_{p 1}\right|^{2}+\left|v_{2} s_{p 2}\right|^{2}+\cdots+\left|v_{n} s_{p n}\right|^{2} \\
& =\left|v_{1}\right|^{2}\left(\left|s_{p 1}\right|^{2}+\left|s_{p 2}\right|^{2}+\cdots+\left|s_{p n}\right|^{2}\right) .
\end{aligned}
$$

This equality together with $v_{i}>v_{i+1}$ shows that

$$
s_{p, i+1}=\cdots=s_{p n}=0 \quad \text { for } \quad p \leq j .
$$

If $q \leq i$, then (2.3.2) again implies $\left|w_{1}\right|^{2} \geq\left|v_{q}\right|^{2}=\left|v_{1}\right|^{2}=\left|w_{1}\right|^{2}$ and so

$$
\begin{aligned}
\left|w_{1}\right|^{2} & =\left|w_{1} t_{1 q}\right|^{2}+\left|w_{2} t_{2 q}\right|^{2}+\cdots+\left|w_{n} t_{n q}\right|^{2} \\
& =\left|w_{1}\right|^{2}\left(\left|t_{1 q}\right|^{2}+\left|t_{2 q}\right|^{2}+\cdots+\left|t_{n q}\right|^{2}\right) .
\end{aligned}
$$

Similarly we obtain that

$$
t_{j+1, q}=\cdots=t_{n q}=0 \quad \text { for } \quad q \leq i
$$

Since the first $j$ row vectors of $S$ and the first $i$ column vectors of $T^{*}$ are linearly independent, we have $i=j$, which becomes $i_{1}$. The assertions (2) and (3) for $S_{1}$ and $T_{1}$ also follow from the unitarity of $S$ and $T$. Therefore the lemma follows from the induction on $n$, since the case of $n=1$ is trivial.

The following lemma is a subtle version of Lemma 2.2 and its proof is technically the same.

LEMMA 2.3. Let $\mathbf{v}$ be decreasing diagonal components given in Lemma 2.2. For two sequences $\left\{S^{k}\right\}$ and $\left\{T^{k}\right\}$ of $\mathrm{U}(n)$ and a sequence of decreasing diagonal components $\left\{\mathbf{d}^{k}\right\}$, suppose that the sequence $\left\{S^{k} \Delta\left(\mathbf{d}^{k}\right) T^{k}\right\}$ converges to $\Delta(\mathbf{v})$. Then

(1) $\left\{\mathbf{d}^{k}\right\}$ converges to $\mathbf{v}$, 
(2) If a pair $(p, q)$ of numbers does not satisfy

$$
i_{1}+i_{2}+\cdots+i_{j}<p, q \leq i_{1}+i_{2}+\cdots+i_{j+1}
$$

for any number $j$ with $0 \leq j<s$, then every sequence $\left\{s_{p q}^{k}\right\}$ (resp. $\left.\left\{t_{p q}^{k}\right\}\right)$ made of $(p, q)$ components of $S^{k}$ (resp. $\left.T^{k}\right)$ converges to zero.

(3) Let $\delta\left(S^{k}\right)$ (resp. $\left.\delta\left(T^{k}\right)\right)$ denote the new matrix made from $S^{k}$ (resp. $\left.T^{k}\right)$ by replacing every $(p, q)$ component described in (2) with zero. Thus $\delta\left(S^{k}\right)$ and $\delta\left(T^{k}\right)$ have the natural decompositions $\delta\left(S^{k}\right)_{1} \dot{+} \cdot \cdots+\delta\left(S^{k}\right)_{s}$ and $\delta\left(T^{k}\right)_{1}+\cdots+\delta\left(T^{k}\right)_{s}$ respectively. Then for any number $j$ with $a_{j} \neq 0$, the sequence $\left\{\delta\left(S^{k}\right)_{j} \delta\left(T^{k}\right)_{j}\right\}$ converges to $E_{i_{j}}$.

Proof. (1) The set of eigen values changes continuously with respect to matrices ([W, Appendix V.4]). By considering the eigen values of $\left(S^{k} \Delta\left(\mathbf{d}^{k}\right) T^{k}\right)^{*}\left(S^{k} \Delta\left(\mathbf{d}^{k}\right) T^{k}\right)$ we know that $\left\{\mathbf{d}^{k}\right\}$ converges to $\mathbf{v}$.

(2) Let $\left(\|A\|=\sum_{i=1}^{n} \sum_{j=1}^{n}\left|a_{i j}\right|^{2}\right)^{1 / 2}$ be the norm of a matrix $A=\left(a_{i j}\right)$. It is clear that $\|S A\|=\|A\|=\|A S\|$ for $S$ in $U(n)$. Set $\mathbf{d}^{k}=\left(d_{1}^{k}, \ldots, d_{n}^{k}\right)$. We may suppose that $v_{1}$ is not zero. By the assumption and (1), given any positive real number $\varepsilon$, there is a number $l$ such that if $k>l$, then we have

$$
\left\|S^{k} \Delta\left(\mathbf{d}^{k}\right) T^{k}-\Delta(\mathbf{v})\right\|<\varepsilon \quad \text { or } \quad\left\|S^{k} \Delta\left(\mathbf{d}^{k}\right)-\Delta(\mathbf{v})\left(T^{k}\right)^{*}\right\|<\varepsilon
$$

and

$$
\left|d_{i}^{k}-v_{i}\right|<\varepsilon \quad \text { for } \quad 1 \leq i \leq n .
$$

Set $S^{k}=\left(s_{p q}^{k}\right)$ and $\left(T^{k}\right)^{*}=\left(t_{p q}^{k}\right)$. Take a number $p$ with $p \leq i_{1}$. Then we have $v_{p}=v_{1} \neq 0$ and

$$
\left|d_{q}^{k} s_{p q}^{k}-v_{p} t_{p q}^{k}\right|<\varepsilon \quad \text { for } \quad 1 \leq q \leq n
$$

It yields

$$
\left|\left(d_{q}^{k} / v_{p}\right) s_{p q}^{k}-t_{p q}^{k}\right|<\varepsilon / v_{p}
$$

and so

$$
\left|t_{p q}^{k}\right|<\left|\left(d_{q}^{k} / v_{p}\right) s_{p q}^{k}\right|+\varepsilon / v_{p}
$$

Hence, we have

$$
\left.1=\sum_{q=1}^{n}\left|t_{p q}^{k}\right|^{2}<\sum_{q=1}^{n}\left(\mid d_{q}^{k} / v_{p}\right) s_{p q}^{k} \mid+\varepsilon / v_{p}\right)^{2}
$$




$$
\begin{aligned}
& \left.\leq \sum_{q=1}^{n}\left(\left|\left(v_{q} / v_{1}\right) s_{p q}^{k}\right|+\mid\left(d_{q}^{k}-v_{q}\right) / v_{1}\right) s_{p q}^{k} \mid+\varepsilon / v_{1}\right)^{2} \\
& <\sum_{q=1}^{n}\left(\left|\left(v_{q} / v_{1}\right) s_{p q}^{k}\right|+2 \varepsilon / v_{1}\right)^{2} \\
& =\sum_{q=1}^{n}\left(v_{q} / v_{1}\right)^{2}\left|s_{p q}^{k}\right|^{2}+\left(4 \varepsilon / v_{1}\right)\left(\sum_{q=1}^{n}\left|\left(v_{q} / v_{1}\right) s_{p q}^{k}\right|\right)+4 n \varepsilon^{2} / v_{1}^{2} \\
& \leq \sum_{q=1}^{i_{1}}\left|s_{p q}^{k}\right|^{2}+\sum_{q=i_{1}+1}^{n}\left(v_{q} / v_{1}\right)^{2}\left|s_{p q}^{k}\right|^{2}+4 n \varepsilon / v_{1}+4 n \varepsilon^{2} / v_{1}^{2} \\
& =1+\sum_{q=i_{1}+1}^{n}\left(-1+\left(v_{q} / v_{1}\right)^{2}\right)\left|s_{p q}^{k}\right|^{2}+4 n \varepsilon / v_{1}+4 n \varepsilon^{2} / v_{1}^{2} .
\end{aligned}
$$

This implies

$$
\sum_{q=i_{1}+1}^{n}\left(1-\left(v_{q} / v_{1}\right)^{2}\right)\left|s_{p q}^{k}\right|^{2}<4 n \varepsilon / v_{1}+4 n \varepsilon^{2} / v_{1}^{2} .
$$

Since $\varepsilon$ can be any positive real number and $\left|v_{q} / v_{1}\right|$ is not bigger than $\left|v_{i_{1}+1} / v_{1}\right|<1$ for $q>i_{1},\left\{s_{p q}^{k}\right\}$ converges to 0 for $p \leq i_{1}$ and $q>i_{1}$ when $k \rightarrow \infty$. Similarly $s_{p q}^{k}$ converges to 0 for such numbers $p$ and $q$. This fact also holds for $T$. Hence (2) is proved by induction on $n$.

(3) It follows from (2) that

$$
\begin{aligned}
\Delta(\mathbf{v}) & =\lim _{k \rightarrow \infty} S^{k} \Delta\left(\mathbf{d}^{k}\right) T^{k} \\
& =\lim _{k \rightarrow \infty} \delta\left(S^{k}\right) \Delta(\mathbf{v}) \delta\left(T^{k}\right) \\
& =\lim _{k \rightarrow \infty} \Delta(\mathbf{v}) \delta\left(S^{k}\right) \delta\left(T^{k}\right) .
\end{aligned}
$$

Since $\mathbf{v}$ is decreasing, $\delta\left(S^{k}\right)_{j} \delta\left(T^{k}\right)_{j}$ must converge to $E_{i_{j}}$ for those numbers $j$ with $a_{j} \neq 0$.

\section{$\S 3$. Homotopy type of $\Omega^{1}$}

In this section we shall study the homology types of $\Omega^{1}$ and $\Sigma^{1}$ in $\operatorname{Hom}\left(\mathbf{C}^{n}, \mathbf{C}^{n}\right)$ for $n \geq 2$. Let $\Omega_{s}^{1}$ (resp. $\Sigma_{s}^{1}$ ) denote the space consisting of all matrices $A=\left(a_{i j}\right)$ such that $A \in \Omega^{1}$ (resp. $A \in \Sigma^{1}$ ) and $\|A\|=1$. Clearly it is a deformation retract of $\Omega^{1}$ (resp. $\left.\Sigma^{1}\right)$. Hence, we study their homotopy types. 
Let $\Delta$ denote the contractible space consisting of all decreasing diagonal components $\mathbf{d}$ such that $d_{n-1}>0$ and $\sum_{i=1}^{n} d_{i}^{2}=1$. In $\Delta$ we consider the subspace consisting of all special diagonal components of the form $\mathbf{d}_{a b}=$ $(a / \sqrt{n-1}, \ldots, a / \sqrt{n-1}, b / \sqrt{n})$, where $a$ and $b$ satisfy $a^{2}+\left(b^{2} / n\right)=1$ and $a / \sqrt{n-1} \geq b / \sqrt{n}$. Note that unless $b=1$, we have $a / \sqrt{n-1}>b / \sqrt{n}$. For a subset $B$ of $[0,1]$ we define $\Delta_{B}$ to be the subset of $\Delta$ consisting of all diagonal components $\mathbf{d}_{a b}$ with $b \in B$.

Lemma 2.1 is a motivation for defining the surjection

$$
\mathcal{H}: \mathrm{SU}(n) \times \Delta \times S^{1} \times \mathrm{SU}(n) \longrightarrow \Omega_{s}^{1}
$$

by $\mathcal{H}\left(S, \mathbf{d}, e^{\sqrt{-1} \theta}, U\right)=S \Delta(\mathbf{d}) I_{-\theta} U$. Here note that given decreasing diagonal components $\mathbf{d}=\left(d_{1}, \ldots, d_{n}\right), S \Delta(\mathbf{d}) I_{-\theta} U \in \Omega_{s}^{1}$ if and only if $\mathbf{d} \in \Delta$. We denote the image $\mathcal{H}\left(\mathrm{SU}(n) \times \Delta_{B} \times S^{1} \times \mathrm{SU}(n)\right)$ by $K(B)$.

THEOREM 3.1. Let $n \geq 2$. There exists a deformation retraction of $\Omega_{s}^{1}$ to $K([0,1])$ whose restriction to $\Sigma_{s}^{1}$ induces a deformation retraction of $\Sigma_{s}^{1}$ to $K(\{0\})$.

Proof. If $n=2$, then it is clear that $\Omega_{s}^{1}$ coincides with $K([0,1])$ and that $\Sigma_{s}^{1}$ coincides with $K(\{0\})$. Thus we may assume that $n \geq 3$. Let $\Delta^{\prime}$ be the set of all diagonal components $\mathbf{f}=\left(f_{1}, \ldots, f_{n-2}, 0,0\right)$ with $f_{1} \geq f_{2} \geq$ $\cdots \geq f_{n-2} \geq 0$ and $\sum_{i=1}^{n-2} f_{i}{ }^{2}=1$. First we shall prove that $\Delta$ is identified with the space $\left(\Delta^{\prime} * \Delta_{[0,1]}\right) \backslash \Delta^{\prime}$, where $\Delta^{\prime} * \Delta_{[0,1]}$ is the join of $\Delta^{\prime}$ and $\Delta_{[0,1]}$ taken on the unit sphere $S^{n-1}$.

For $\mathbf{d}=\left(d_{1}, \ldots, d_{n}\right)$ of $\Delta \backslash \Delta_{[0,1]}$, there exist uniquely determined $\mathbf{f}$, $\mathbf{d}_{a b}$ and $t$ with $1>t>0$ such that if we set $\mathbf{d}^{\prime}=t \mathbf{f}+(1-t) \mathbf{d}_{a b}$, then $\mathbf{d}=\mathbf{d}^{\prime} /\left\|\mathbf{d}^{\prime}\right\|$. In fact, let $t \mathbf{f}+(1-t) \mathbf{d}_{a b}=c\left(s \mathbf{f}^{\prime}+(1-s) \mathbf{d}_{a^{\prime} b^{\prime}}\right)$ with $c>0$. Then

$$
\begin{gathered}
(1-t) a=c(1-s) a^{\prime}, \quad(1-t) b=c(1-s) b^{\prime}, \\
(1-t)^{2}\left(a^{2}+\left(b^{2} / n\right)\right)=c^{2}(1-s)^{2}\left(a^{\prime 2}+\left(b^{\prime 2} / n\right)\right) .
\end{gathered}
$$

This yields $1-t=c(1-s)$. Hence $a=a^{\prime}$ and $b=b^{\prime}$. So we have $t \mathbf{f}=c s \mathbf{f}^{\prime}$ and $t=c s$. Thus we obtain that $t=s, a=a^{\prime}, b=b^{\prime}, \mathbf{f}=\mathbf{f}^{\prime}$ and $c=1$.

Next we show the existence of $\mathbf{f}, \mathbf{d}_{a b}$ and $t$. By using the equation $\mathbf{d}=\mathbf{d}^{\prime} /\left\|\mathbf{d}^{\prime}\right\|$, we obtain

$$
\left\|\mathbf{d}^{\prime}\right\| d_{n-1}=(1-t) a / \sqrt{n-1}, \quad\left\|\mathbf{d}^{\prime}\right\| d_{n}=(1-t) b / \sqrt{n}
$$


and

$$
\left\|\mathbf{d}^{\prime}\right\|^{2}\left((n-1) d_{n-1}^{2}+d_{n}^{2}\right)=(1-t)^{2}\left(a^{2}+\left(b^{2} / n\right)\right)=(1-t)^{2} .
$$

For simplicity, set $u=\left((n-1) d_{n-1}^{2}+d_{n}^{2}\right)^{1 / 2}>0$. It must be that $a=$ $\sqrt{n-1} d_{n-1} / u$ and $b=\sqrt{n} d_{n} / u$ with $a^{2}+\left(b^{2} / n\right)=1$ and $a / \sqrt{n-1} \geq$ $b / \sqrt{n}$, and that $\mathbf{f}$ and $t$ satisfy the equation

$$
\mathbf{d}=\left(1 /\left\|\mathbf{d}^{\prime}\right\|\right) t \mathbf{f}+\left((1-t) /\left\|\mathbf{d}^{\prime}\right\|\right) \mathbf{d}_{a b}=(u t /(1-t)) \mathbf{f}+u \mathbf{d}_{a b} .
$$

Therefore, for $\mathbf{d}$ of $\Delta \backslash \Delta_{[0,1]}$ we define $a$ and $b$ as above, and $\mathbf{f}$ and $t$ so that they satisfy $\mathbf{f}=\left(\mathbf{d}-u \mathbf{d}_{a b}\right) /\left\|\mathbf{d}-u \mathbf{d}_{a b}\right\|$ and $u t /(1-t)=\left\|\mathbf{d}-u \mathbf{d}_{a b}\right\|$. By definition, it is easy to see that $f_{n-1}=f_{n}=0,\left\|\mathbf{d}-u \mathbf{d}_{a b}\right\|>0$ and $0<t<1$.

In the following we represent $\mathbf{d}$ in $\Delta$ as $\left(t \mathbf{f}+(1-t) \mathbf{d}_{a b}\right) /\left\|t \mathbf{f}+(1-t) \mathbf{d}_{a b}\right\|$, where $\mathbf{d} \in \Delta_{[0,1]}$ if and only if $t=0$. Now we define the deformation retraction $r_{\lambda}$ of $\Delta$ to $\Delta_{[0,1]}$ with $r=\operatorname{id}_{\Delta}$ by

$r_{\lambda}(\mathbf{d})=\left((1-\lambda)\left(t \mathbf{f}+(1-t) \mathbf{d}_{a b}\right)+\lambda \mathbf{d}_{a b}\right) /\left\|(1-\lambda)\left(t \mathbf{f}+(1-t) \mathbf{d}_{a b}\right)+\lambda \mathbf{d}_{a b}\right\|$.

It has the property that if $d_{i}=d_{j}$, then the $i$-th and the $j$-th components of $r_{\lambda}(\mathbf{d})$ denoted by $d_{i}^{\lambda}$ and $d_{j}^{\lambda}$ respectively coincide with each other. In fact, for the case $i \leq j \leq n-1$ this follows from $\mathbf{f}=\left(\mathbf{d}-u \mathbf{d}_{a b}\right) /\left\|\mathbf{d}-u \mathbf{d}_{a b}\right\|$ and for the case $i \leq n-1$ and $j=n$, we have $d_{i}=d_{i+1}=\cdots=d_{n}$ and so $a / \sqrt{n-1}=b / \sqrt{n}$. This yields $f_{i}=f_{i+1}=\cdots=f_{n}$ and so $d_{i}^{\lambda}=d_{i+1}^{\lambda}=\cdots=d_{n}^{\lambda}$.

Now we define the deformation retraction $R_{\lambda}$ of $\Omega_{s}^{1}$ to $K([0,1])$, whose restriction of $\Sigma_{s}^{1}$ induces a deformation retraction of $\Sigma_{s}^{1}$ to $K(\{0\})$. We always consider the representation of a matrix $A$ of $\Omega_{s}^{1}$ as $A=S \Delta(\mathbf{d}) I_{-\theta} U$, where $S, T \in \mathrm{SU}(n)$. Then define $R_{\lambda}$ by $R_{\lambda}(A)=S \Delta\left(r_{\lambda}(\mathbf{d})\right) I_{-\theta} U$. This is well defined and continuous as is seen below. Let $A=S^{\prime} \Delta(\mathbf{d}) I_{-\theta} U^{\prime}$. If $d_{i}=d_{j}$, then $d_{i}^{\lambda}=d_{j}^{\lambda}$. Furthermore, the matrices $\left(S^{\prime}\right)^{*} S$ and $I_{-\theta} U\left(U^{\prime}\right)^{*} I_{\theta}$ belong to $\mathrm{SU}(n)$ and satisfy the properties stated in Lemma 2.2, since $\left(S^{\prime}\right)^{*} S \Delta(\mathbf{d}) I_{-\theta} U\left(U^{\prime}\right)^{*} I_{\theta}=\Delta(\mathbf{d})$. Hence, it follows that $\left(S^{\prime}\right)^{*} S \Delta\left(r_{\lambda}(\mathbf{d})\right) \times$ $I_{-\theta} U\left(U^{\prime}\right)^{*} I_{\theta}=\Delta\left(r_{\lambda}(\mathbf{d})\right)$. This implies that $R_{\lambda}(A)$ does not depend on the choice of $S$ and $U$. It is easy to see that $R_{\lambda}(A)$ keeps $\Sigma_{s}^{1}$ and that $R_{1}$ maps $\Sigma_{s}^{1}$ onto $K(\{0\})$.

For the proof of continuity, take a sequence $\left\{A^{k}\right\}$ of $\Omega_{s}^{1}$ with representation $A^{k}=S^{k} \Delta\left(\mathbf{d}^{k}\right) I_{-\theta_{k}} U^{k}$ as in Lemma 2.3 and a sequence $\left\{\lambda_{m}\right\}$ such 
that $\lim _{k \rightarrow \infty} A^{k}=A$ and $\lim _{m \rightarrow \infty} \lambda_{m}=\lambda$. Then $\left\{\mathbf{d}^{k}\right\}$ converges to $\mathbf{d}$ by Lemma 2.3 (1). Since

$$
\lim _{k \rightarrow \infty} S^{*} S^{k} \Delta\left(\mathbf{d}^{k}\right) I_{-\theta_{k}} U^{k} U^{*} I_{\theta}-\Delta(\mathbf{d})
$$

it follows that $S^{*} S^{k}$ and $I_{-\theta_{k}} U^{k} U^{*} I_{\theta}$ satisfy the properties of Lemma 2.3, which induce $\delta\left(S^{*} S^{k}\right)$ and $\delta\left(I_{-\theta_{k}} U^{k} U^{*} I_{\theta}\right)$. Therefore, we have

$$
\begin{aligned}
\lim _{k \rightarrow \infty, m \rightarrow \infty} & S^{*} S^{k} \Delta\left(r_{\lambda_{m}}\left(\mathbf{d}^{k}\right)\right) I_{-\theta_{k}} U^{k} U^{*} I_{\theta} \\
= & \lim _{k \rightarrow \infty, m \rightarrow \infty} \delta\left(S^{*} S^{k}\right) \Delta\left(r_{\lambda_{m}}\left(\mathbf{d}^{k}\right)\right) \delta\left(I_{-\theta_{k}} U^{k} U^{*} I_{\theta}\right) \\
= & \lim _{k \rightarrow \infty, m \rightarrow \infty} \Delta\left(r_{\lambda_{m}}\left(\mathbf{d}^{k}\right)\right) \delta\left(S^{*} S^{k}\right) \delta\left(I_{-\theta_{k}} U^{k} U^{*} I_{\theta}\right) \\
= & \Delta\left(r_{\lambda}(\mathbf{d})\right) .
\end{aligned}
$$

Thus (3.1.2) proves that $\lim _{k \rightarrow \infty, m \rightarrow \infty} R_{\lambda_{m}}\left(A^{k}\right)=R_{\lambda}(A)$.

In the following we shall prove that $K([0,1])$ is the space stated in Theorem 1 (1) in Introduction.

We begin by proving that the restriction of $\mathcal{H}$ to $\mathrm{SU}(n) \times \Delta_{(0,1)} \times$ $S^{1} \times \mathrm{SU}(n)$ onto $K((0,1))$ is a fibre bundle. Let $\mathcal{H}\left(S, \mathbf{d}_{a b}, e^{\sqrt{-1} \theta}, U\right)=$ $\mathcal{H}\left(S^{\prime}, \mathbf{d}_{a b}, e^{\sqrt{-1} \theta}, U^{\prime}\right)$. Then $\left(S^{\prime}\right)^{*} S \Delta\left(\mathbf{d}_{a b}\right) I_{-\theta} U\left(U^{\prime}\right)^{*} I_{\theta}=\Delta\left(\mathbf{d}_{a b}\right)$. Вy we have that $\left(S^{\prime}\right)^{*} S$ and $I_{-\theta} U\left(U^{\prime}\right)^{*} I_{\theta}$ have the decompositions $S_{1} \dot{+}\left(z_{1}\right)$ and $U_{1} \dot{+}\left(z_{2}\right)$ respectively with $S_{1} U_{1}=E_{n-1}$ and $z_{1} z_{2}=1$. Hence we have $\left(S^{\prime}\right)^{*} S I_{-\theta} U\left(U^{\prime}\right)^{*} I_{\theta}=E_{n}$, that is, $S I_{-\theta} U=S^{\prime} I_{-\theta} U^{\prime}$ and $S \mathbf{e}_{n}=S^{\prime}\left(S_{1} \dot{+}\right.$ $\left.\left(z_{1}\right)\right) \mathbf{e}_{n}=z_{1} S^{\prime} \mathbf{e}_{n}$, where $\mathbf{e}_{n}={ }^{t}(0, \ldots, 0,1)$. This observation enables us to define the surjections,

$$
\begin{aligned}
& P: \mathrm{SU}(n) \times \Delta_{(0,1)} \times S^{1} \times \mathrm{SU}(n) \longrightarrow \mathbf{C P}^{n-1} \times \Delta_{(0,1)} \times S^{1} \times \mathrm{SU}(n), \\
& H: \mathbf{C P}^{n-1} \times \Delta_{(0,1)} \times S^{1} \times \mathrm{SU}(n) \longrightarrow K((0,1))
\end{aligned}
$$

by $P\left(S, \mathbf{d}_{a b}, e^{\sqrt{-1} \theta}, U\right)=\left(\left[S \mathbf{e}_{n}\right], \mathbf{d}_{a b}, e^{\sqrt{-1} \theta}, I_{\theta} S I_{-\theta} U\right)$ and $\mathcal{H} \mid \mathrm{SU}(n) \times$ $\Delta_{(0,1)} \times S^{1} \times \mathrm{SU}(n)=H \circ P$, where [*] refers to the element of $\mathbf{C P}^{n-1}$ represented by $*$. The precise description of $H$ is as follows. Let $\mathbf{v}$ be an element of $\mathbf{C P}^{n-1}$ represented by a vector $\mathbf{s}$ with length 1 . Find a matrix $S$ of $\mathrm{SU}(n)$ with $S \mathbf{e}_{n}=\mathbf{s}$ (this notation will be often used below without stating it explicitly). Then we know that

$$
H\left(\mathbf{v}, \mathbf{d}_{a b}, e^{\sqrt{-1} \theta}, U\right)=S \Delta\left(\mathbf{d}_{a b}\right) S^{*} I_{-\theta} U .
$$


In fact, it does not depend on the choice of $\mathbf{s}$ and $S$, because a direct calculation shows

$$
S \Delta(x, \ldots, x, y) S^{*}=x E_{n}+(y-x)\left(s_{i} \bar{s}_{j}\right)
$$

and we have

$$
\begin{aligned}
H \circ P\left(S, \mathbf{d}_{a b}, e^{\sqrt{-1} \theta}, U\right) & =H\left(\mathbf{v}, \mathbf{d}_{a b}, e^{\sqrt{-1} \theta}, I_{\theta} S I_{-\theta} U\right) \\
& =S \Delta\left(\mathbf{d}_{a b}\right) S^{*} I_{-\theta}\left(I_{\theta} S I_{-\theta} U\right) \\
& =S \Delta\left(\mathbf{d}_{a b}\right) I_{-\theta} U \\
& =\mathcal{H}\left(S, \mathbf{d}_{a b}, e^{\sqrt{-1} \theta}, U\right) .
\end{aligned}
$$

Here we note that $H$ is naturally extended to the continuous surjection $\widetilde{H}$ of $\mathrm{OC}\left(\mathbf{C P}^{n-1}\right) \times S^{1} \times \mathrm{SU}(n)$ onto $K((0,1])$ by setting $\widetilde{H}\left(*, e^{\sqrt{-1} \theta}, U\right)=$ $(1 / \sqrt{n}) I_{-\theta} U$, where $*$ is the cone point, since we have

$$
\begin{aligned}
\lim _{b \rightarrow 1} H\left(\mathbf{v}, \mathbf{d}_{a b}, e^{\sqrt{-1} \theta}, U\right) & =\lim _{b \rightarrow 1} S \Delta\left(\mathbf{d}_{a b}\right) S^{*} I_{-\theta} U \\
& =S(1 / \sqrt{n}) E_{n} S^{*} I_{-\theta} U=(1 / \sqrt{n}) I_{-\theta} U
\end{aligned}
$$

which does not depend on the vector $\mathbf{v}$. Here note that the point $\left(\mathbf{v}, \mathbf{d}_{a b}\right)$ corresponds to the point $\left(\mathbf{v},\left(1-b^{2}\right)^{1 / 2}\right)$ in $\mathrm{OC}\left(\mathbf{C P}^{n-1}\right)=\mathbf{C P}^{n-1} \times$ $[0,1) / \mathbf{C P}^{n-1} \times 0$.

We define the other map

$$
P_{\Sigma}: \mathrm{SU}(n) \times \Delta_{(0,1 / 2)} \times S^{1} \times \mathrm{SU}(n) \longrightarrow \mathbf{C P}^{n-1} \times \Delta_{(0,1 / 2)} \times S^{1} \times \mathrm{SU}(n)
$$

by $P_{\Sigma}\left(S, \mathbf{d}_{a b}, e^{\sqrt{-1} \theta}, U\right)=\left(\left[S \mathbf{e}_{n}\right], \mathbf{d}_{a b}, e^{\sqrt{-1} \theta}, \mathrm{SU}\right)$. This map induces the surjection

$$
H_{\Sigma}: \mathbf{C P}^{n-1} \times \Delta_{(0,1 / 2)} \times S^{1} \times \mathrm{SU}(n) \longrightarrow K((0,1 / 2))
$$

defined by

$$
H_{\Sigma}\left(\mathbf{v}, \mathbf{d}_{a b}, e^{\sqrt{-1} \theta}, U\right)=S \Delta\left(\mathbf{d}_{a b}\right) I_{-\theta} S^{*} U
$$

so that $\mathcal{H} \mid \mathrm{SU}(n) \times \Delta_{(0,1 / 2)} \times S^{1} \times \mathrm{SU}(n)=H_{\Sigma} \circ P_{\Sigma}$, where $S$ is a matrix of $\mathrm{SU}(n)$ with $\left[S \mathbf{e}_{n}\right]=\mathbf{v}$. In fact, this map is well defined, since 
$S \Delta\left(\mathbf{d}_{a b}\right) I_{-\theta} S^{*} U=S \Delta\left(\mathbf{d}_{a b}\right) S^{*} S I_{-\theta} S^{*} U$, and $S \Delta\left(\mathbf{d}_{a b}\right) S^{*}$ and $S I_{-\theta} S^{*}$ depend only on $\mathbf{v}$ by (3.3). Then we have

$$
\begin{aligned}
H_{\Sigma} \circ P_{\Sigma}\left(S, \mathbf{d}_{a b}, e^{\sqrt{-1} \theta}, U\right) & =H_{\Sigma}\left(\left[S \mathbf{e}_{n}\right], \mathbf{d}_{a b}, e^{\sqrt{-1} \theta}, \mathrm{SU}\right) \\
& =S \Delta\left(\mathbf{d}_{a b}\right) I_{-\theta} U \\
& =\mathcal{H}\left(S, \mathbf{d}_{a b}, e^{\sqrt{-1} \theta}, U\right) .
\end{aligned}
$$

Now $H_{\Sigma}$ is naturally extended to the continuous surjection

$$
\widetilde{H}_{\Sigma}: \mathbf{C P}^{n-1} \times \operatorname{Int} D_{1 / 2}^{2} \times \mathrm{SU}(n) \longrightarrow K([0,1 / 2))
$$

defined by $\widetilde{H}_{\Sigma}\left(\mathbf{v}, b e^{\sqrt{-1} \theta}, U\right)=H_{\Sigma}\left(\mathbf{v}, \mathbf{d}_{a b}, e^{\sqrt{-1} \theta}, U\right)$ for $0<b<1 / 2$ and $\widetilde{H}_{\Sigma}(\mathbf{v}, \mathbf{0}, U)=S \Delta(1 / \sqrt{n-1}, \ldots, 1 / \sqrt{n-1}, 0) S^{*} U$, since we have

$$
\begin{aligned}
\lim _{b \rightarrow 0} H_{\Sigma}\left(\mathbf{v}, \mathbf{d}_{a b}, e^{\sqrt{-1} \theta}, U\right) & =\lim _{b \rightarrow 0} S \Delta\left(\mathbf{d}_{a b}\right) I_{-\theta} S^{*} U \\
& =S \Delta(1 / \sqrt{n-1}, \ldots, 1 / \sqrt{n-1}, 0) S^{*} U
\end{aligned}
$$

Lemma 3.5. (1) The map $\widetilde{H}: \mathrm{OC}\left(\mathbf{C P}^{n-1}\right) \times S^{1} \times \mathrm{SU}(n) \rightarrow K((0,1])$ is a continuous bijection.

(2) The map $\widetilde{H}_{\Sigma}: \mathbf{C P}^{n-1} \times \operatorname{Int} D_{1 / 2}^{2} \times \mathrm{SU}(n) \rightarrow K([0,1 / 2))$ is a continuous bijection.

Proof. (1) Let $A$ be a matrix of $K((0,1])$, which is represented as $S \Delta\left(\mathbf{d}_{a b}\right) I_{-\theta} U$ with $S, U \in \mathrm{SU}(n)$. We show that the inverse $H_{1}$ of $\widetilde{H}$ is given by

$$
\begin{array}{ll}
H_{1}(A)=\left(\left[S \mathbf{e}_{n}\right], \mathbf{d}_{a b}, e^{\sqrt{-1} \theta}, I_{\theta} S I_{-\theta} U\right) & \text { for } \quad 0<b<1, \\
H_{1}(A)=\left(*, e^{\sqrt{-1} \theta}, I_{\theta} S I_{-\theta} U\right) & \text { for } \quad b=1 .
\end{array}
$$

First we see that $H_{1}$ is well defined. By Lemma $2.1, \mathbf{d}_{a b}$ is determined by $A$. Let $S^{\prime} \Delta\left(\mathbf{d}_{a b}\right) I_{-\theta} U^{\prime}$ be another representation. Then it follows from Lemma 2.2 that $S I_{-\theta} U=S^{\prime} I_{-\theta} U^{\prime}$, and $\left[S \mathbf{e}_{n}\right]=\left[S^{\prime} \mathbf{e}_{n}\right]$ for $0<b<1$. Let us see that it is actually the inverse of $\widetilde{H}$. In fact, for $0<b<1$, we have

$$
\begin{aligned}
\widetilde{H} \circ H_{1}(A) & =\widetilde{H}\left(\left[S \mathbf{e}_{n}\right], \mathbf{d}_{a b}, e^{\sqrt{-1} \theta}, I_{\theta} S I_{-\theta} U\right) \\
& =S \Delta\left(\mathbf{d}_{a b}\right) S^{*} I_{-} I_{\theta} S I_{-\theta} U \\
& =A
\end{aligned}
$$


and for $b=1$, we have

$$
\begin{aligned}
\widetilde{H} \circ H_{1}(A) & =\widetilde{H}\left(*, e^{\sqrt{-1} \theta}, I_{\theta} S I_{-\theta} U\right) \\
& =(1 / \sqrt{n}) I_{-\theta} I_{\theta} S I_{-\theta} U \\
& =S(1 / \sqrt{n}) E_{n} I_{-\theta} U \\
& =A .
\end{aligned}
$$

We have, inversely, for $0<b<1$

$$
\begin{aligned}
H_{1} \circ \widetilde{H}\left(\mathbf{v}, \mathbf{d}_{a b}, e^{\sqrt{-1} \theta}, U\right) & =H_{1}\left(S \Delta\left(\mathbf{d}_{a b}\right) S^{*} I_{-\theta} U\right) \\
& =H_{1}\left(S \Delta\left(\mathbf{d}_{a b}\right) I_{-\theta} I_{\theta} S^{*} I_{-\theta} U\right) \\
& =\left(\left[S \mathbf{e}_{n}\right], \mathbf{d}_{a b}, e^{\sqrt{-1} \theta}, I_{\theta} S I_{-\theta} I_{\theta} S^{*} I_{-\theta} U\right) \\
& =\left(\left[S \mathbf{e}_{n}\right], \mathbf{d}_{a b}, e^{\sqrt{-1} \theta}, U\right) .
\end{aligned}
$$

Similarly, for $b=1$, we see that $H_{1} \circ \widetilde{H}\left(*, e^{\sqrt{-1} \theta}, U\right)=\left(*, e^{\sqrt{-1} \theta}, U\right)$.

(2) A matrix $A$ of $K([0,1 / 2))$ is represented as $S \Delta\left(\mathbf{d}_{a b}\right) I_{-\theta} U$ as above and the inverse $\left(H_{\Sigma}\right)_{1}$ of $\widetilde{H}_{\Sigma}$ is given by

$$
\left.\left(H_{\Sigma}\right)_{1}(A)=\left(\left[S \mathbf{e}_{n}\right]\right), b e^{\sqrt{-1} \theta}, \mathrm{SU}\right) .
$$

It follows from Lemma 2.2 that this is well defined. In fact, let $A=S^{\prime} \Delta\left(\mathbf{d}_{a b}\right) I_{-\theta^{\prime}} U^{\prime}$ be another representation of $A$. Then we have $S^{*} S^{\prime} \Delta\left(\mathbf{d}_{a b}\right) I_{-\theta^{\prime}} U^{\prime} U^{*} I_{\theta}=\Delta\left(\mathbf{d}_{a b}\right)$. We can represent as $S^{*} S^{\prime}=S_{1} \dot{+}\left(z_{1}\right)$ and $I_{-\theta^{\prime}} U^{\prime} U^{*} I_{\theta}=U_{1} \dot{+}\left(z_{2}\right)$, that is, $U^{\prime} U^{*}=U_{1} \dot{+}\left(z_{2}\right)$ with $S_{1} U_{1}=E_{n-1}$, and $z_{1} z_{2}=1$ by Lemma 2.2 for $b>0$ and by $z_{1} \operatorname{det} S_{1}=z_{2} \operatorname{det} S_{2}=1$ for $b=0$. Hence, we have $S^{*} S^{\prime} U^{\prime} U^{*}=E_{n}$ and so $S U=S^{\prime} U^{\prime} .\left(H_{\Sigma}\right)_{1}$ is actually the inverse of $\widetilde{H}_{\Sigma}$, since we have

$$
\begin{aligned}
\widetilde{H}_{\Sigma} \circ\left(H_{\Sigma}\right)_{1}(A) & =\widetilde{H}_{\Sigma}\left(\left[S \mathbf{e}_{n}\right], b e^{\sqrt{-1} \theta}, S U\right) \\
& =S \Delta\left(\mathbf{d}_{a b}\right) I_{-\theta} S^{*} S U \\
& =A
\end{aligned}
$$

and

$$
\begin{aligned}
\left(H_{\Sigma}\right)_{1} \circ \widetilde{H}_{\Sigma}\left(\mathbf{v}, b e^{\sqrt{-1} \theta}, U\right) & =\left(H_{\Sigma}\right)_{1}\left(S \Delta\left(\mathbf{d}_{a b}\right) I_{-\theta} S^{*} U\right) \\
& =\left(\left[S \mathbf{e}_{n}\right], b e^{\sqrt{-1} \theta}, U\right) .
\end{aligned}
$$


Consequently we have two bijections of $\mathbf{C P}^{n-1} \times \Delta_{(0,1 / 2)} \times S^{1} \times \mathrm{SU}(n)$ onto $K((0,1 / 2))$ by $H$ and $H_{\Sigma}$. Here recall the matrix $G\left(\mathbf{v}, e^{\sqrt{-1} \theta}\right)=$ $I_{\theta}\left(E_{n}+\left(e^{-\sqrt{-1} \theta}-1\right)\left(s_{i} \bar{s}_{j}\right)\right)$ in Introduction, which is equal to $I_{\theta} S I_{-\theta} S^{*}$ by (3.3) for all $S$ with $\left[S \mathbf{e}_{n}\right]=\mathbf{v}$. Let us determine the map $H^{-1} \circ H_{\Sigma}$ by using (3.2), (3.4) and Lemma 3.5. We have

$$
\begin{aligned}
H^{-1} \circ H_{\Sigma}\left(\mathbf{v}, \mathbf{d}_{a b}, e^{\sqrt{-1} \theta}, U\right) & =H^{-1}\left(S \Delta\left(\mathbf{d}_{a b}\right) I_{-\theta} S^{*} U\right) \\
& =\left(\mathbf{v}, \mathbf{d}_{a b}, e^{\sqrt{-1} \theta}, I_{\theta} S I_{-\theta} S^{*} U\right) \\
& =\left(\mathbf{v}, \mathbf{d}_{a b}, e^{\sqrt{-1} \theta}, G\left(\mathbf{v}, e^{\sqrt{-1} \theta}\right) U\right)
\end{aligned}
$$

It is easy to see that $H^{-1} \circ H_{\Sigma} \mid \mathbf{C P}^{n-1} \times \Delta_{(0,1 / 2)} \times S^{1} \times \mathrm{SU}(n)$ is a homeomorphism.

THEOREM 3.7. Let $n \geq 2$. Under the notation in Introduction, the space $K([0,1])$ is homeomorphic to $\mathbf{C P}^{n-1} \times \operatorname{Int} D_{1 / 2}^{2} \times \mathrm{SU}(n) \cup_{g} \mathrm{OC}\left(\mathbf{C P}^{n-1}\right)$ $\times S^{1} \times \mathrm{SU}(n)$ and the space $K(\{0\})$ is homeomorphic to $\mathbf{C P}^{n-1} \times\{\mathbf{0}\} \times$ $\mathrm{SU}(n)$.

Proof. We define the map $j_{n}: \mathbf{C P}^{n-1} \times \operatorname{Int} D_{1 / 2}^{2} \times \mathrm{SU}(n) \cup_{g} \mathrm{OC}\left(\mathbf{C P}^{n-1}\right)$ $\times S^{1} \times \mathrm{SU}(n) \rightarrow K([0,1])$ by $j_{n}\left(\mathbf{v}, b e^{\sqrt{-1} \theta}, U\right)=\widetilde{H}_{\Sigma}\left(\mathbf{v}, b e^{\sqrt{-1} \theta}, U\right)$ for $0 \leq$ $b<1 / 2$ and $j_{n}\left(\mathbf{v},\left(1-b^{2}\right)^{1 / 2}, e^{\sqrt{-1} \theta}, U\right)=\widetilde{H}\left(\mathbf{v}, \mathbf{d}_{a b}, e^{\sqrt{-1} \theta}, U\right)$ for $0<b \leq$ 1. It follows from Lemma 3.5 and (3.6) that $j_{n}$ is well defined and is a continuous bijection. Since $\mathbf{C P}^{n-1} \times \operatorname{Int} D_{1 / 2}^{2} \times \mathrm{SU}(n) \cup_{g} \mathrm{OC}\left(\mathbf{C P}^{n-1}\right) \times$ $S^{1} \times \mathrm{SU}(n)$ is compact, we have that $j_{n}$ is a homeomorphism. Furthermore, $j_{n}$ maps $\mathbf{C P}^{n-1} \times \mathbf{0} \times \mathrm{SU}(n)$ onto $K(\{0\})$.

Proof of Theorem 1(1). The assertion follows from Theorems 3.1 and 3.7 .

Remark 3.8. Let $\mathbf{v}=\left[S \mathbf{e}_{n}\right]$ as above. The kernel of $\widetilde{H}_{\Sigma}(\mathbf{v}, \mathbf{0}, U)$ is generated by $U^{*} S \mathbf{e}_{n}$ and the orthogonal complement of its image is generated by $S \mathbf{e}_{n}$.

\section{$\S 4$. Structure of the fibre bundle $\mathrm{SU}(n+1)$ over $\mathrm{SU}(n+1) / \mathrm{SU}(n)$}

In this section let $n \geq 1$. In contrast to the canonical basis $\left\{\mathbf{e}_{1}, \ldots, \mathbf{e}_{n}\right\}$ of $\mathbf{C}^{n}$, we write the canonical basis of $\mathbf{C}^{n+1}$ by $\left\{\mathbf{e}_{1}^{\prime}, \ldots, \mathbf{e}_{n+1}^{\prime}\right\}$. Let $E_{n+1}$ be the unit matrix of rank $n+1$. We shall consider the fibre bundle $\pi$ : $\mathrm{SU}(n+1) \rightarrow \mathrm{SU}(n+1) / \mathrm{SU}(n) \times(1) \cong S^{2 n+1}$ and specify its structure. In 
this paper a point of $S^{2 n+1}$ will be written as $\mathbf{z}={ }^{t}\left(x_{1}, \ldots, x_{n}, z_{n+1}\right)$ with $\mathbf{x}={ }^{t}\left(x_{1}, \ldots, x_{n}\right) \in \mathbf{C}^{n}$ and $z_{n+1}=b e^{\sqrt{-1} \theta}$. Let $S_{\mathcal{R}}$ and $S_{\Sigma}$ be the subsets of $S^{2 n+1}$ consisting of all points $z$ such that $0<b \leq 1$ and $0 \leq b<1 / 2$ respectively.

For a point $\mathbf{z}$ of $S_{\mathcal{R}}$ with $0<b \leq 1$, we define the matrix $r(\mathbf{z})$ of $\mathrm{SU}(n+1)$ so that

$$
\begin{gathered}
r(\mathbf{z})\left(\mathbf{e}_{n+1}^{\prime}\right)=e^{-\sqrt{-1} \theta} \mathbf{z}, \\
r(\mathbf{z})\left(\mathbf{z}-z_{n+1} \mathbf{e}_{n+1}^{\prime}\right)=b \mathbf{z}-e^{\sqrt{-1} \theta} \mathbf{e}_{n+1}^{\prime},
\end{gathered}
$$

(4.1-(iii)) if $0<b<1$, then $r(\mathbf{z})$ is the identity on the orthogonal complement of the subspace generated by the two vectors $\mathbf{e}_{n+1}^{\prime}$ and $\mathbf{z}-z_{n+1} \mathbf{e}_{n+1}^{\prime}$ over $\mathbf{C}$ and if $b=1$, then $r(z)=E_{n+1}$.

For a point $\mathbf{z}$ of $S_{\Sigma}$ with $0 \leq b<1 / 2$, we define the matrix $r_{\Sigma}(\mathbf{z})$ of $\mathrm{SU}(n+1)$ so that

$$
\begin{gathered}
r_{\Sigma}(\mathbf{z})\left(\mathbf{e}_{n+1}^{\prime}\right)=\mathbf{z} \\
r_{\Sigma}(\mathbf{z})\left(\mathbf{z}-z_{n+1} \mathbf{e}_{n+1}^{\prime}\right)=\bar{z}_{n+1} \mathbf{z}-\mathbf{e}_{n+1}^{\prime},
\end{gathered}
$$

(4.2-(iii)) $\quad r_{\Sigma}(\mathbf{z})$ is the identity on the orthogonal complement of the subspace generated by the two vectors $\mathbf{e}_{n+1}^{\prime}$ and $\mathbf{z}-z_{n+1} \mathbf{e}_{n+1}^{\prime}$ over $\mathbf{C}$.

The explicit formulas of the matrices $r(\mathbf{z})$ and $r_{\Sigma}(\mathbf{z})$ are as follows:

$$
r(\mathbf{z})=\left(\begin{array}{cc}
R(\mathbf{z}) & e^{-\sqrt{-1} \theta} \mathbf{x} \\
-e^{\sqrt{-1} \theta}\left({ }^{t} \overline{\mathbf{x}}\right) & b
\end{array}\right) \text { and } r_{\Sigma}(\mathbf{z})=\left(\begin{array}{cc}
R_{\Sigma}(\mathbf{x}) & \mathbf{x} \\
-{ }^{t} \overline{\mathbf{x}} & b e^{\sqrt{-1} \theta}
\end{array}\right)
$$

where the $(i, j)$ components of $R(\mathbf{z})$ and $R_{\Sigma}(\mathbf{z})$ are $\delta_{i j}-x_{i} \bar{x}_{j} /(1+b)$ and $\delta_{i j}-x_{i} \bar{x}_{j}\left(\left(1-b e^{-\sqrt{-1} \theta}\right) /\left(1-b^{2}\right)\right)$ respectively.

LEMmA 4.3. The determinants of $r(\mathbf{z})$ and $r_{\Sigma}(\mathbf{z})$ are equal to 1. 
Proof. First we show $\operatorname{det}(r(\mathbf{z}))=1$. For $b \neq 1$, let $\mathbf{f}_{1}, \ldots, \mathbf{f}_{n-1}$ denote vectors such that $\left(\mathbf{f}_{1}, \ldots, \mathbf{f}_{n-1}, \mathbf{e}_{n+1}^{\prime},\left(\mathbf{z}-z_{n+1} \mathbf{e}_{n+1}^{\prime}\right) /\|x\|\right)$ is an orthonormal basis. Then by definition we have

$$
\begin{aligned}
r(\mathbf{z})\left(\mathbf{f}_{1}, \ldots, \mathbf{f}_{n-1}, \mathbf{e}_{n+1}^{\prime},\left(\mathbf{z}-z_{n+1} \mathbf{e}_{n+1}^{\prime}\right) /\|\mathbf{x}\|\right) \\
=\left(\mathbf{f}_{1}, \ldots, \mathbf{f}_{n-1}, e^{-\sqrt{-1} \theta} \mathbf{z},\left(b \mathbf{z}-e^{\sqrt{-1} \theta} \mathbf{e}_{n+1}^{\prime}\right) /\|\mathbf{x}\|\right)
\end{aligned}
$$

Hence,

$$
\begin{aligned}
& \|\mathbf{x}\|^{2} \operatorname{det}(r(\mathbf{z})) \\
& =\operatorname{det}\left(\left(\mathbf{f}_{1}, \ldots, \mathbf{f}_{n-1}, \mathbf{e}_{n+1}^{\prime}, \mathbf{z}-z_{n+1} \mathbf{e}_{n+1}^{\prime}\right)^{*}\right. \\
& \left.\quad \times\left(\mathbf{f}_{1}, \ldots, \mathbf{f}_{n-1}, e^{-\sqrt{-1} \theta} \mathbf{z}, b \mathbf{z}-e^{\sqrt{-1} \theta} \mathbf{e}_{n+1}^{\prime}\right)\right) \\
& =\operatorname{det}\left(E_{n-1}+\left({ }^{t}\left(\overline{\mathbf{e}_{n+1}^{\prime}, \mathbf{z}-z_{n+1} \mathbf{e}_{n+1}^{\prime}}\right)\left(e^{-\sqrt{-1} \theta} \mathbf{z}, b \mathbf{z}-e^{\sqrt{-1} \theta} \mathbf{e}_{n+1}^{\prime}\right)\right)\right) \\
& \left.=\operatorname{det}\left(\frac{t}{\left(\mathbf{e}_{n+1}^{\prime}, \mathbf{z}-z_{n+1} \mathbf{e}_{n+1}^{\prime}\right.}\right)\left(e^{-\sqrt{-1} \theta} \mathbf{z}, b \mathbf{z}-e^{\sqrt{-1} \theta} \mathbf{e}_{n+1}^{\prime}\right)\right) \\
& =\operatorname{det}\left(\begin{array}{c}
\left(-\|\mathbf{x}\|^{2}\right) e^{\sqrt{-1} \theta} \\
\quad b\|\mathbf{x}\|^{2}
\end{array}\right) \\
& =\|\mathbf{x}\|^{2}\left(b^{2}+\|\mathbf{x}\|^{2}\right) \\
& =\|\mathbf{x}\|^{2} .
\end{aligned}
$$

Next let us show that $\operatorname{det}\left(r_{\Sigma}(\mathbf{z})\right)=1$. Take an orthonormal basis $\left(\mathbf{f}_{1}, \ldots, \mathbf{f}_{n-1}, \mathbf{e}_{n+1}^{\prime},\left(\mathbf{z}-z_{n+1} \mathbf{e}_{n+1}^{\prime}\right) /\|\mathbf{x}\|\right)$. Then by definition we have

$$
\begin{gathered}
r_{\Sigma}(\mathbf{z})\left(\mathbf{f}_{1}, \ldots, \mathbf{f}_{n-1}, \mathbf{e}_{n+1}^{\prime},\left(\mathbf{z}-z_{n+1} \mathbf{e}_{n+1}^{\prime}\right) /\|\mathbf{x}\|\right) \\
=\left(\mathbf{f}_{1}, \ldots, \mathbf{f}_{n-1}, \mathbf{z},\left(\bar{z}_{n+1} \mathbf{z}-\mathbf{e}_{n+1}^{\prime}\right) /\|\mathbf{x}\|\right) .
\end{gathered}
$$

Hence,

$$
\begin{aligned}
& \|\mathbf{x}\|^{2} \operatorname{det}\left(r_{\Sigma}(\mathbf{z})\right) \\
& =\operatorname{det}\left(\left(\mathbf{f}_{1}, \ldots, \mathbf{f}_{n-1}, \mathbf{e}_{n+1}^{\prime}, \mathbf{z}-z_{n+1} \mathbf{e}_{n+1}^{\prime}\right)^{*}\right. \\
& \left.\quad \times\left(\mathbf{f}_{1}, \ldots, \mathbf{f}_{n-1}, \mathbf{z}, \bar{z}_{n+1} \mathbf{z}-\mathbf{e}_{n+1}^{\prime}\right)\right) \\
& \left.=\operatorname{det}\left(E_{n-1}+{ }^{t}\left(\overline{\mathbf{e}_{n+1}^{\prime}, \mathbf{z}-z_{n+1} \mathbf{e}_{n+1}^{\prime}}\right)\left(\mathbf{z}, \bar{z}_{n+1} \mathbf{z}-\mathbf{e}_{n+1}^{\prime}\right)\right)\right)
\end{aligned}
$$




$$
\begin{aligned}
& \left.=\operatorname{det}\left(\overline{\left(\mathbf{e}_{n+1}^{\prime}, \mathbf{z}-z_{n+1} \mathbf{e}_{n+1}^{\prime}\right.}\right)\left(\mathbf{z}, \bar{z}_{n+1} \mathbf{z}-\mathbf{e}_{n+1}^{\prime}\right)\right) \\
& =\operatorname{det}\left(\begin{array}{cc}
b e^{\sqrt{-1} \theta} & -\|\mathbf{x}\|^{2} \\
\|\mathbf{x}\|^{2} & b e^{-\sqrt{-1} \theta}\|\mathbf{x}\|^{2}
\end{array}\right) \\
& =\|\mathbf{x}\|^{2} .
\end{aligned}
$$

LEMMA 4.4. For a point $\mathbf{z}={ }^{t}\left(x_{1}, \ldots, x_{n}\right)$, be $\left.{ }^{\sqrt{-1} \theta}\right)$ of $S^{2 n+1}$ with $0<$ $b<1 / 2$, set $\mathbf{s}=\mathbf{x} /\|\mathbf{x}\|$ and let $S$ be a matrix of $\mathrm{SU}(n)$ with $S \mathbf{e}_{n}=\mathbf{s}$. Then we have

$$
r(\mathbf{z})^{-1} r_{\Sigma}(\mathbf{z})=S I_{-\theta} S^{*} \dot{+}\left(e^{\sqrt{-1} \theta}\right) .
$$

Proof. Let $T$ be the matrix $(S \dot{+}(1))^{*} r(\mathbf{z})^{-1} r_{\Sigma}(\mathbf{z})(S \dot{+}(1))\left(I_{\theta} \dot{+}(1)\right)$. Then we have

$$
\text { (1) } \begin{aligned}
T\left(\mathbf{e}_{n+1}^{\prime}\right) & =e^{\sqrt{-1} \theta} \mathbf{e}_{n+1}^{\prime} \\
\text { (2) } T\left(\|\mathbf{x}\| \mathbf{e}_{n}^{\prime}\right) & =(S \dot{+}(1))^{*} r(\mathbf{z})^{-1} r_{\Sigma}(\mathbf{z})(S \dot{+}(1))\left(\|\mathbf{x}\| e^{\sqrt{-1} \theta} \mathbf{e}_{n}^{\prime}\right) \\
& =(S \dot{+}(1))^{*} r(\mathbf{z})^{-1} r_{\Sigma}(\mathbf{z})\left(e^{\sqrt{-1} \theta}\left(\mathbf{z}-z_{n+1} \mathbf{e}_{n+1}^{\prime}\right)\right) \\
& =(S \dot{+}(1))^{*} r(\mathbf{z})^{-1}\left(b \mathbf{z}-e^{\sqrt{-1} \theta} \mathbf{e}_{n+1}^{\prime}\right) \\
& =(S+(1))^{*}\left(\mathbf{z}-z_{n+1} \mathbf{e}_{n+1}^{\prime}\right) \\
& =\|\mathbf{x}\| \mathbf{e}_{n}^{\prime} .
\end{aligned}
$$

Since $(S+(1)) \mathbf{e}_{i}^{\prime}(i=1, \ldots, n-1)$ belong to the orthogonal complement of the space generated by $\mathbf{e}_{n+1}^{\prime}$ and $\mathbf{z}-z_{n+1} \mathbf{e}_{n+1}^{\prime}$, we obtain by (4.1-(iii)) and (4.2-(iii) that

$$
\text { (3) } T \mathbf{e}_{i}^{\prime}=\mathbf{e}_{i}^{\prime} \quad(i=1, \ldots, n-1) .
$$

Therefore, it follows that $T=E_{n} \dot{+}\left(e^{\sqrt{-1} \theta}\right)$.

For a matrix $M \in \mathrm{SU}(n+1)$, let $M \mathbf{e}_{n+1}^{\prime}$ be written as $\mathbf{z}={ }^{t}\left(x_{1}, \ldots, x_{n}\right.$, $\left.z_{n+1}\right)$ with $\mathbf{x}(M)={ }^{t}\left(x_{1}, \ldots, x_{n}\right)$ and $z_{n+1}=b e^{\sqrt{-1} \theta}$. If $0<b \leq 1$, then $r(\mathbf{z})^{-1} M \mathbf{e}_{n+1}^{\prime}=r(\mathbf{z})^{-1} \mathbf{z}=e^{\sqrt{-1} \theta} \mathbf{e}_{n+1}^{\prime}$ and $r(\mathbf{z})^{-1} M$ is written as $I_{-\theta} U(M)+\left(e^{\sqrt{-1} \theta}\right)$ by some matrix $U(M)$ of $\mathrm{SU}(n)$. If $0 \leq b<1 / 2$, then $r_{\Sigma}(\mathbf{z})^{-1} M \mathbf{e}_{n+1}^{\prime}=r_{\Sigma}(\mathbf{z})^{-1} \mathbf{z}=\mathbf{e}_{n+1}^{\prime}$ and $r_{\Sigma}(\mathbf{z})^{-1} M$ is written as $U_{\Sigma}(M) \dot{+}$ 
(1) by some matrix $U_{\Sigma}(M)$ of $\mathrm{SU}(n)$. If $\|x(M)\|$ is not 0 , then set $\mathbf{s}(M)=$ $\mathbf{x}(M) /\|\mathbf{x}(M)\|$. We define the trivializations

$$
\begin{aligned}
& t_{\mathcal{R}}: \pi^{-1}\left(S_{\mathcal{R}}\right) \longrightarrow \operatorname{Int} D_{1}^{2 n} \times S^{1} \times \mathrm{SU}(n) \quad \text { and } \\
& t_{\Sigma}: \pi^{-1}\left(S_{\Sigma}\right) \longrightarrow S^{2 n-1} \times \operatorname{Int} D_{1 / 2}^{2} \times \mathrm{SU}(n)
\end{aligned}
$$

of $\pi^{-1}\left(S_{\mathcal{R}}\right)$ and $\pi^{-1}\left(S_{\Sigma}\right)$ by $t_{\mathcal{R}}(M)=\left(x(M), e^{\sqrt{-1} \theta}, U(M)\right)$ and $t_{\Sigma}(M)=$ $\left(\mathbf{s}(M), b e^{\sqrt{-1} \theta}, U_{\Sigma}(M)\right)$ respectively. It is not difficult to see that they are really trivializations. From now on, when a vector $\mathbf{s}$ representing $[\mathbf{s}]$ is specified, the matrix $I_{\theta} S I_{-\theta} S^{*}$ is denoted by $G\left(\mathbf{s}, e^{\sqrt{-1} \theta}\right)$ in place of $G\left([\mathbf{s}], e^{\sqrt{-1} \theta}\right)$.

Proposition 4.6. If $0<b<1 / 2$ then we have

$$
t_{\mathcal{R}} \circ t_{\Sigma}^{-1}\left(\mathbf{s}, b e^{\sqrt{-1} \theta}, U_{\Sigma}\right)=\left(\left(1-b^{2}\right)^{1 / 2} \mathbf{s}, e^{\sqrt{-1} \theta}, G\left(\mathbf{s}, e^{\sqrt{-1} \theta}\right) U_{\Sigma}\right) .
$$

Proof. There exists a matrix $M$ of $\mathrm{SU}(n+1)$ such that $\mathbf{s}=\mathbf{s}(M)$, $U_{\Sigma}=U_{\Sigma}(M)$ and ${ }^{t} \mathbf{z}={ }^{t}\left(M \mathbf{e}_{n+1}^{\prime}\right)=\left({ }^{t} \mathbf{x}(M), b e^{\sqrt{-1} \theta}\right)$. By definition, we have $t_{\Sigma}^{-1}\left(\mathbf{s}(M), b e^{\sqrt{-1} \theta}, U_{\Sigma}(M)\right)=M=r_{\Sigma}(\mathbf{z})\left(U_{\Sigma}(M)+(1)\right)$. Again by definition of $U(M)$, we have

$$
I_{-\theta} U(M) \dot{+}\left(e^{\sqrt{-1} \theta}\right)=r(\mathbf{z})^{-1} M=r(\mathbf{z})^{-1} r_{\Sigma}(\mathbf{z})\left(U_{\Sigma}(M) \dot{+}(1)\right)
$$

and so

$$
U(M) \dot{+}(1)=\left(I_{\theta} \dot{+}\left(e^{-\sqrt{-1} \theta}\right)\right) r(\mathbf{z})^{-1} r_{\Sigma}(\mathbf{z})\left(U_{\Sigma}(M) \dot{+}(1)\right) .
$$

By Lemma 4.4 this is equal to

$$
\begin{gathered}
\left(I_{\theta} \dot{+}\left(e^{-\sqrt{-1} \theta}\right)\right)\left(S I_{-\theta} S^{*} \dot{+}\left(e^{\sqrt{-1} \theta}\right)\right)\left(U_{\Sigma}(M) \dot{+}(1)\right) \\
=\left(I_{\theta} S I_{-\theta} S^{*} U_{\Sigma}(M)\right) \dot{+}(1) \\
=G\left(\mathbf{s}(M), e^{\sqrt{-1} \theta}\right) U_{\Sigma}(M) \dot{+}(1) .
\end{gathered}
$$

Hence, $t_{\mathcal{R}}(M)=\left(\mathbf{x}(M), e^{\sqrt{-1} \theta}, G\left(\mathbf{s}(M), e^{\sqrt{-1} \theta}\right) U_{\Sigma}(M)\right)$ with $\mathbf{x}(M)=$ $\left(1-b^{2}\right)^{1 / 2} \mathbf{s}(M)$.

Let $\widetilde{g}$ be the diffeomorphism

$$
\begin{aligned}
\tilde{g}: S^{2 n-1} \times \operatorname{Int}\left(D_{1 / 2}^{2} \backslash\{0\}\right) \times \mathrm{SU}(n) & \\
& \longrightarrow \operatorname{Int}\left(D_{1}^{2 n} \backslash D_{\sqrt{3} / 2}^{2 n}\right) \times S^{1} \times \mathrm{SU}(n)
\end{aligned}
$$


defined by $\widetilde{g}\left(\mathbf{s}, b e^{\sqrt{-1} \theta}, U_{\Sigma}\right)=\left(\left(1-b^{2}\right)^{1 / 2} \mathbf{s}, e^{\sqrt{-1} \theta}, G\left(\mathbf{s}, e^{\sqrt{-1} \theta}\right) U_{\Sigma}\right)(0<b<$ $1 / 2)$. Let $S^{2 n-1} \times \operatorname{Int} D_{1 / 2}^{2} \times \mathrm{SU}(n) \cup_{g} \operatorname{Int} D_{1}^{2 n} \times S^{1} \times \mathrm{SU}(n)$ denote the space obtained by pasting the two spaces written above by $\widetilde{g}$. Then we can define the diffeomorphism $k: \mathrm{SU}(n+1) \rightarrow S^{2 n-1} \times \operatorname{Int} D_{1 / 2}^{2} \times \mathrm{SU}(n) \cup_{\tilde{g}} \operatorname{Int} D_{1}^{2 n} \times$ $S^{1} \times \mathrm{SU}(n)$ by

$$
k(M)= \begin{cases}\left(\mathbf{x}(M), e^{\sqrt{-1} \theta}, U(M)\right) & \text { for } \quad 0<b \leq 1 \\ \left(\mathbf{s}(M), b e^{\sqrt{-1} \theta}, U_{\Sigma}(M)\right) & \text { for } \quad 0 \leq b<1 / 2 .\end{cases}
$$

The map $\pi^{\prime}: S^{2 n-1} \times \operatorname{Int} D_{1 / 2}^{2} \times \mathrm{SU}(n) \cup_{g}^{\sim} \operatorname{Int} D_{1}^{2 n} \times S^{1} \times \mathrm{SU}(n) \rightarrow S^{2 n+1}$ defined by $\pi^{\prime}\left(\mathbf{x}, e^{\sqrt{-1} \theta}, U\right)=\left(\mathbf{x},\left(1-\|\mathbf{x}\|^{2}\right)^{1 / 2} e^{\sqrt{-1} \theta}\right)$ for $0<b \leq 1$ and $\pi^{\prime}\left(\mathbf{s}, b e^{\sqrt{-1} \theta}, U_{\Sigma}\right)=\left(\left(1-b^{2}\right)^{1 / 2} \mathbf{s}, b e^{\sqrt{-1} \theta}\right)$ for $0 \leq b<1 / 2$ becomes a principal bundle with fibre $\mathrm{SU}(n)$. Then the following proposition follows from the arguments above.

Proposition 4.9. Let $n \geq 1$. The map $k$ above gives a $C^{\infty}$ bundle map of the principal bundle $\pi: \mathrm{SU}(n+1) \rightarrow \mathrm{SU}(n+1) / \mathrm{SU}(n) \times(1) \cong S^{2 n+1}$ to the principal bundle $\pi^{\prime}: S^{2 n-1} \times \operatorname{Int} D_{1 / 2}^{2} \times \mathrm{SU}(n) \cup_{g} \operatorname{Int} D_{1}^{2 n} \times S^{1} \times \mathrm{SU}(n) \rightarrow$ $S^{2 n+1}$.

\section{$\S 5$. Homotopy type of $\Omega^{10}$}

We first review the homotopy type of $\Sigma^{10}$ in the context of Sections 3 and 4 . Let $\pi_{1}^{2}$ be the canonical forgetting map of $J^{2}(n, n)$ onto $J^{1}(n, n)$. Now we see what fibre bundle the restriction $\pi_{1}^{2} \mid \Sigma^{10}: \Sigma^{10} \rightarrow \Sigma^{1}$ is. When $\left(\pi_{1}^{2}\right)^{-1}\left(\Sigma^{1}\right)$ is identified with $\Sigma^{1} \times \operatorname{Hom}\left(\mathbf{C}^{n} \circ \mathbf{C}^{n}, \mathbf{C}^{n}\right)$, we have two line bundles $\mathbf{K}$ and $\mathbf{Q}$ over $\Sigma^{1}$ defined by

$$
\mathbf{K}=\left\{(\alpha, \mathbf{k}) \mid \alpha \in \Sigma^{1}, \mathbf{k} \in \operatorname{Ker} \alpha\right\}
$$

and

$$
\mathbf{Q}=\left\{(\alpha, \mathbf{v}) \mid \alpha \in \Sigma^{1}, \mathbf{v} \in \operatorname{Cok} \alpha\right\}
$$

respectively. Then we have the following exact sequence of vector bundles over $\Sigma^{1}$ :

$$
0 \longrightarrow \mathbf{K} \longrightarrow \Sigma^{1} \times \mathbf{C}^{n} \stackrel{h}{\longrightarrow} \Sigma^{1} \times \mathbf{C}^{n} \longrightarrow \mathbf{Q} \longrightarrow 0
$$

where $h$ is the fibrewise homomorphism defined by $h(\alpha, \mathbf{x})=(\alpha, \alpha(\mathbf{x}))$. Consider the map $C: \Sigma^{1} \rightarrow \mathbf{C P}^{n-1}$ defined as $C(\alpha)$ being the line orthogonal to $\operatorname{Im}(\alpha)$ in $\mathbf{C}^{n}$. Then $C_{1}(\mathbf{K})=C_{1}(\mathbf{Q})=C^{*}\left(c_{1}\right)$, where $c_{1}$ is the 
first Chern class of the canonical line bundle over $\mathbf{C} \mathbf{P}^{n-1}$. It is known that the normal bundle of $\Sigma^{1}$ in $J^{1}(n, n)$ is equivalent to $\operatorname{Hom}(\mathbf{K}, \mathbf{Q}$ ) (see [L, p.11, 2. Proof of Proposition 2] and [Bo, p.50, Lemma 7.13 and Theorem 7.14]). Since $C_{1}(\operatorname{Hom}(\mathbf{K}, \mathbf{Q}))=C_{1}(\mathbf{Q})-C_{1}(\mathbf{K})=0$, this normal bundle is trivial. Restricting the map $\widetilde{H}_{\Sigma}$ to $\mathbf{C P}^{n-1} \times \mathbf{0} \times \mathrm{SU}(n)$ in Section 3 , we have an embedding of $\mathbf{C P}^{n-1} \times \mathbf{0} \times \mathrm{SU}(n)$ into $\Sigma^{1}$ inducing a homotopy equivalence. The composition of $C$ and $\widetilde{H}_{\Sigma} \mid \mathbf{C P}^{n-1} \times \mathbf{0} \times \mathrm{SU}(n)$ coincides with the canonical projection of $\mathbf{C P}^{n-1} \times \mathbf{0} \times \mathrm{SU}(n)$ onto $\mathbf{C P}^{n-1}$, since $C \circ \widetilde{H}_{\Sigma}\left(\mathbf{v}, \mathbf{0}, U_{\Sigma}\right)=C\left(S \Delta\left(\mathbf{d}_{10}\right) S^{*} U_{\Sigma}\right)=\left[S \mathbf{e}_{n}\right]=\mathbf{v}$. This implies $\left(\widetilde{H}_{\Sigma} \mid \mathbf{C P}^{n-1} \times \mathbf{0} \times \mathrm{SU}(n)\right)^{*}\left(C_{1}(\mathbf{Q})\right)=c_{1} \times 1$. We define the fibrewise homomorphism $r$ of $\Sigma^{1} \times \operatorname{Hom}\left(\mathbf{C}^{n} \bigcirc \mathbf{C}^{n}, \mathbf{C}^{n}\right)$ onto $\operatorname{Hom}(\mathbf{K} \bigcirc \mathbf{K}, \mathbf{Q})$ over $\Sigma^{1}$ by $r(\alpha, \beta)=\operatorname{pr} \circ \beta \mid \operatorname{Ker}(\alpha) \bigcirc \operatorname{Ker}(\alpha)$, where pr denotes the projection of $\mathbf{C}^{n}$ onto $\operatorname{Cok}(\alpha)$. Let $\mathfrak{R}$ be the subspace of $\operatorname{Hom}(\mathbf{K} \bigcirc \mathbf{K}, \mathbf{Q})$ consisting of all isomorphisms. By the definition of $\Sigma^{10}$ we know that $\Sigma^{10}$ coincides with $r^{-1}(\mathfrak{R})$. Since $C_{1}(\operatorname{Hom}(\mathbf{K} \bigcirc \mathbf{K}, \mathbf{Q}))=-2 C_{1}(\mathbf{K})+C_{1}(\mathbf{Q})=-C_{1}(\mathbf{K})$, $\operatorname{Hom}(\mathbf{K} \bigcirc \mathbf{K}, \mathbf{Q})$ is equivalent to $\operatorname{Hom}(\mathbf{K}, \mathbf{C})$ as vector bundles, and there is an orientation reversing bundle map between the associated sphere bundles $S(\operatorname{Hom}(\mathbf{K}, \mathbf{C}))$ and $S(\mathbf{K})$. Hence the fibre bundle $\Sigma^{10}$ over $\Sigma^{1}$ is homotopy equivalent to the $S^{1}$-bundle $S^{2 n-1} \times \mathbf{0} \times \mathrm{SU}(n)$ over $\mathbf{C P}^{n-1} \times \mathbf{0} \times \mathrm{SU}(n)$ induced from the $S^{1}$-bundle of $S^{2 n-1}$ over $\mathbf{C P}^{n-1}$ associated with $c_{1}$ of $H^{2}\left(\mathbf{C P}^{n-1} ; \mathbf{Z}\right)$. Furthermore $\Sigma^{10}$ has $S^{2 n-1} \times \mathbf{0} \times \mathrm{SU}(n)$ as its deformation retract.

In $\Omega^{10}, \Sigma^{0} \times \operatorname{Hom}\left(\mathbf{C}^{n} \bigcirc, \mathbf{C}^{n}, \mathbf{C}^{n}\right)$ over $\Sigma^{0}$ has a contractible fibre. Hence by the arguments above, $\Omega^{10}$ has, as its deformation retract, the subspace which is the total space of the above $S^{1}$-bundle over $\mathbf{C P}^{n-1} \times \operatorname{Int} D_{1 / 2}^{2} \times$ $\mathrm{SU}(n) \cup_{g} \mathrm{OC}\left(\mathbf{C P}^{n-1}\right) \times S^{1} \times \mathrm{SU}(n)$ except for over $\{*\} \times S^{1} \times \mathrm{SU}(n)$ with $*$ being the cone point of $\mathrm{OC}\left(\mathbf{C P}^{n-1}\right)$. It is nothing but $S^{2 n-1} \times \operatorname{Int} D_{1 / 2}^{2} \times$ $\mathrm{SU}(n) \cup_{g} \operatorname{Int} D_{1}^{2 n} \times S^{1} \times \mathrm{SU}(n)$. Hence it follows from Proposition 4.9 that $\Omega^{10}$ is homotopy equivalent to $\mathrm{SU}(n+1)$. This is an intuitive proof of Theorem $1(2)$.

Now we shall specify the embedding

$$
h: S^{2 n-1} \times \operatorname{Int} D_{1 / 2}^{2} \times \mathrm{SU}(n) \bigcup_{\widetilde{g}} \operatorname{Int} D_{1}^{2 n} \times S^{1} \times \mathrm{SU}(n) \longrightarrow \Omega^{10} .
$$

For a point $\left(\mathbf{x}, e^{\sqrt{-1} \theta}, U\right)$ of $\operatorname{Int} D_{1}^{2 n} \times S^{1} \times \mathrm{SU}(n)$, we define the map $\beta\left(\mathbf{x}, e^{\sqrt{-1} \theta}, U\right): \mathbf{C}^{n} \bigcirc \mathbf{C}^{n} \rightarrow \mathbf{C}^{n}$ by

$(5.1) \beta\left(\mathbf{x}, e^{\sqrt{-1} \theta}, U\right)(\mathbf{a}, \mathbf{b})$ 


$$
\begin{aligned}
& \quad=\left\{{ }^{t} \mathbf{a}^{t}\left(G\left(\mathbf{s}, e^{\sqrt{-1} \theta}\right)^{*} U\right) \bar{S} \Delta(0, \ldots, 0,\|\mathbf{x}\|) S^{*}\left(G\left(\mathbf{s}, e^{\sqrt{-1} \theta}\right)^{*} U\right) \mathbf{b}\right\} \mathbf{s} \\
& \text { for } \quad \mathbf{x} \neq \mathbf{0} \quad \text { and } \\
& \beta\left(\mathbf{0}, e^{\sqrt{-1} \theta}, U\right)(\mathbf{a}, \mathbf{b})=\mathbf{0},
\end{aligned}
$$

where if $\|\mathbf{x}\| \neq 0$, then $\mathbf{s}=\mathbf{x} /\|\mathbf{x}\|$ and $S \mathbf{e}_{n}=\mathbf{s}$. The matrix

$$
{ }^{t}\left(G\left(\mathbf{s}, e^{\sqrt{-1} \theta}\right)^{*} U\right) \bar{S} \Delta(0, \ldots, 0,\|\mathbf{x}\|) S^{*}\left(G\left(\mathbf{s}, e^{\sqrt{-1} \theta}\right)^{*} U\right)
$$

is equal to

$$
\begin{gathered}
{ }^{t} U I_{-\theta} \bar{S} I_{\theta}{ }^{t} S \bar{S} \Delta(0, \ldots, 0,\|\mathbf{x}\|) S^{*} S I_{\theta} S^{*} I_{-\theta} U \\
={ }^{t} U I_{-\theta} \bar{S} I_{\theta} \Delta(0, \ldots, 0,\|\mathbf{x}\|) I_{\theta} S^{*} I_{-\theta} U \\
={ }^{t} U I_{-\theta}\left(e^{2 \sqrt{-1} \theta}\|\mathbf{x}\|\left(\bar{s}_{i} \bar{s}_{j}\right)\right) I_{-\theta} U
\end{gathered}
$$

For a point $\left(\mathbf{s}, b e^{\sqrt{-1} \theta}, U_{\Sigma}\right)$ of $S^{2 n-1} \times \operatorname{Int} D_{1 / 2}^{2} \times \mathrm{SU}(n)$, we define the map $\beta_{\Sigma}\left(\mathbf{s}, b e^{\sqrt{-1} \theta}, U_{\Sigma}\right): \mathbf{C}^{n} \bigcirc \mathbf{C}^{n} \rightarrow \mathbf{C}^{n}$ by

$$
\beta_{\Sigma}\left(\mathbf{s}, b e^{\sqrt{-1} \theta}, U_{\Sigma}\right)(\mathbf{a}, \mathbf{b})=\left\{{ }^{t} \mathbf{a}^{t} U_{\Sigma} \bar{S} \Delta\left(0, \ldots, 0,\left(1-b^{2}\right)^{1 / 2}\right) S^{*} U_{\Sigma} \mathbf{b}\right\} \mathbf{s}
$$

which is equal to

$$
\left\{{ }^{t} \mathbf{a}^{t} U_{\Sigma}\left(\left(1-b^{2}\right)^{1 / 2}\left(\bar{s}_{i} \bar{s}_{j}\right)\right) U_{\Sigma} \mathbf{b}\right\} \mathbf{s}
$$

If $0<b<1 / 2$, then we have that $\beta\left(\mathbf{x}, e^{\sqrt{-1} \theta}, U\right)=\beta_{\Sigma}\left(\mathbf{s}, b e^{\sqrt{-1} \theta}, U_{\Sigma}\right)$, since $U=G\left(\mathbf{s}, e^{\sqrt{-1} \theta}\right) U_{\Sigma}=I_{\theta} S I_{-\theta} S^{*} U_{\Sigma}$, where $\|\mathbf{x}\|=\left(1-b^{2}\right)^{1 / 2}$ by definition. Hence, $\beta$ and $\beta_{\Sigma}$ define the well-defined map of $S^{2 n-1} \times \operatorname{Int} D_{1 / 2}^{2} \times \operatorname{SU}(n) \cup_{g}$ Int $D_{1}^{2 n} \times S^{1} \times \mathrm{SU}(n)$ to $\operatorname{Hom}\left(\mathbf{C}^{n} \bigcirc \mathbf{C}^{n}, \mathbf{C}^{n}\right)$.

The motivation for the definition above is the facts that when $b=0$, we have $\widetilde{H}_{\Sigma}\left([\mathbf{s}], \mathbf{0}, U_{\Sigma}\right)=S \Delta\left(\mathbf{d}_{10}\right) I_{-\theta} S^{*} U_{\Sigma}=S \Delta\left(\mathbf{d}_{10}\right) S^{*} U_{\Sigma}$ and that its kernel vector is $U_{\Sigma}{ }^{*} S \mathbf{e}_{n}$ and its cokernel vector is s. Hence, if $b=0$, then we should have that $\beta_{\Sigma}\left(\mathbf{s}, \mathbf{0}, U_{\Sigma}\right)\left(U_{\Sigma}{ }^{*} \mathbf{e}_{n}, U_{\Sigma}{ }^{*} S \mathbf{e}_{n}\right)=\mathbf{s}$. If $b=1$, then $\widetilde{H}\left(\mathbf{0}, e^{\sqrt{-1} \theta}, U\right)=(1 / \sqrt{n}) I_{-\theta} U$ and we must require $\beta\left(\mathbf{0}, e^{\sqrt{-1} \theta}, U\right)$ to be the null-homomorphism.

From now on, we often use the notation $\widetilde{H}\left(\mathbf{x}, e^{\sqrt{-1} \theta}, U\right)\left(\operatorname{resp} . \widetilde{H}\left(\mathbf{0}, e^{\sqrt{-1} \theta}\right.\right.$, $U)$ ) in place of $H\left([\mathbf{s}], \mathbf{d}_{a b}, e^{\sqrt{-1} \theta}, U\right)$ (resp. $\left.(1 / \sqrt{n}) I_{-\theta} U\right)$ for $0<b<1$ (resp. $b=1)$ and $\widetilde{H}_{\Sigma}\left(\mathbf{s}, b e^{\sqrt{-1} \theta}, U_{\Sigma}\right)$ in place of $\widetilde{H}_{\Sigma}\left([\mathbf{s}], b e^{\sqrt{-1} \theta}, U_{\Sigma}\right)$ for simplicity, when a vector $\mathbf{x}$ or $\mathbf{s}$ representing $[\mathbf{x}]$ or $[\mathbf{s}]$ is specified respectively. Then 
the map $h$ is defined by

$$
\begin{aligned}
& \left(h \mid \operatorname{Int} D_{1}^{2 n} \times S^{1} \times \mathrm{SU}(n)\right)\left(\mathbf{x}, e^{\sqrt{-1} \theta}, U\right) \\
& =\left(\widetilde{H}\left(\mathbf{x}, e^{\sqrt{-1} \theta}, U\right), \beta\left(\mathbf{x}, e^{\sqrt{-1} \theta}, U\right)\right) \quad(0<b \leq 1), \\
& \left(h \mid S^{2 n-1} \times \operatorname{Int} D_{1 / 2}^{2} \times \mathrm{SU}(n)\right)\left(\mathbf{s}, b e^{\sqrt{-1} \theta}, U_{\Sigma}\right) \\
& =\left(\widetilde{H}_{\Sigma}\left(\mathbf{s}, b e^{\sqrt{-1} \theta}, U_{\Sigma}\right), \beta_{\Sigma}\left(\mathbf{s}, b e^{\sqrt{-1} \theta}, U_{\Sigma}\right)\right) \quad(0 \leq b<1 / 2) \text {. }
\end{aligned}
$$

We have the following proposition by the definition of $h$ together with the observation above.

Proposition 5.4. The map $h$ is a topological embedding $(n \geq 2)$.

We define the topological embedding $i_{n}: \mathrm{SU}(n+1) \rightarrow \Omega^{10}$ as follows.

$$
\begin{aligned}
& \text { For } n=1, i_{n}(M)=\left(b e^{\sqrt{-1} \theta}, \overline{\mathbf{x}}\right), \\
& \text { for } n \geq 2 \\
& i_{n}(M)=h \circ k(M) \\
& =\left\{\begin{array}{r}
\left(\widetilde{H}\left(\mathbf{x}(M), e^{\sqrt{-1} \theta}, U(M)\right), \beta\left(\mathbf{x}(M), e^{\sqrt{-1} \theta}, U(M)\right)\right) \\
(0<b \leq 1) \\
\left(\widetilde{H}_{\Sigma}\left(\mathbf{s}(M), b e^{\sqrt{-1} \theta}, U_{\Sigma}(M)\right), \beta_{\Sigma}\left(\mathbf{s}(M), b e^{\sqrt{-1} \theta}, U_{\Sigma}(M)\right)\right) \\
(0 \leq b<1 / 2) .
\end{array}\right.
\end{aligned}
$$

THEOREM 5.5. The map $i_{n}$ is a topological embedding and the image of $i_{n}$ is a deformation retract of $\Omega^{10}$.

Proof. We only need to prove the second assertion. The case $n=1$ is easy to prove. Hence, we assume $n \geq 2$. By Proposition 4.9 and the definition of $i_{n}$, the image of $i_{n}$ coincides with that of $h$. By Theorem 3.1, it is enough to construct a deformation retraction of $\left(\pi_{1}^{2} \mid \Omega^{10}\right)^{-1}(K([0,1]))$ to the image of $h$. We identify an element $\beta$ of $\operatorname{Hom}\left(\mathbf{C}^{n} \bigcirc \mathbf{C}^{n}, \mathbf{C}^{n}\right)$ with the $n$-tuple $\left(B_{1}, \ldots, B_{n}\right)$ of symmetric $n$-matrices. Then the norm $\|\beta\|$ is defined to be $\sum_{i=1}^{n}\left\|B_{i}\right\|$.

We first consider the homotopy $h_{\lambda}$ of $\left(\pi_{1}^{2} \mid \Omega^{10}\right)^{-1}(K([0,1]))$ defined as follows. For an element $(\alpha, \beta)$ of $\left(\pi_{1}^{2} \mid \Omega^{10}\right)^{-1}(K(\{b\}))$, we set

$h_{\lambda}(\alpha, \beta)$ 


$$
=\left\{\begin{array}{cc}
\left(\alpha,\left((1-\lambda)+\lambda\left(1-b^{2}\right)^{1 / 2}\right)\left(\|\beta\|-2\left(1-b^{2}\right)^{1 / 2}\right)(\beta /\|\beta\|)\right. \\
\left.+2\left(1-b^{2}\right)^{1 / 2}(\beta /\|\beta\|)\right) & \text { if }\|\beta\| \geq 2\left(1-b^{2}\right)^{1 / 2} \text { and }\|\beta\| \neq 0, \\
(\alpha, \beta) & \text { if }\|\beta\| \leq 2\left(1-b^{2}\right)^{1 / 2}
\end{array}\right.
$$

It is easy to see that the image of $h_{1}$ coincides with $\left(\pi_{1}^{2} \mid \Omega^{10}\right)^{-1}(K([0,1))) \cup$ $K(\{1\}) \times\{\mathbf{0}\}$.

Next we construct a deformation retraction $R_{\lambda}$ of $\left(\pi_{1}^{2} \mid \Omega^{10}\right)^{-1}(K([0,1)))$ $\cup K(\{1\}) \times\{\mathbf{0}\}$ to the image of $h$. Take an element $(\alpha, \beta)$ of $\left(\pi_{1}^{2} \mid \Omega^{10}\right)^{-1}($ $K(\{b\}))$ such that $\alpha$ is written as $\widetilde{H}\left([\mathbf{x}], \mathbf{d}_{a b}, e^{\sqrt{-1} \theta}, U\right)$ with $\|\mathbf{x}\|=$ $\left(1-b^{2}\right)^{1 / 2}$ for $0<b<1$ or $\widetilde{H}_{\Sigma}\left([\mathbf{s}], b e^{\sqrt{-1} \theta}, U_{\Sigma}\right)$ for $0 \leq b<1 / 2$. Let $\widetilde{K}_{\alpha}$ be the subspace generated by $U^{*} G\left(\mathbf{s}, e^{\sqrt{-1} \theta}\right) \mathbf{x}$ for $0<b<1$ and the subspace generated by $U_{\Sigma}^{*} \mathbf{S}$ for $0 \leq b<1 / 2$. Let $\widetilde{Q}_{\alpha}$ be the subspace generated by $\mathbf{x}$ or s for $0 \leq b<1$. Let $\widetilde{\mathbf{K}}$ and $\widetilde{\mathbf{Q}}$ be the complex line bundles over $K([0,1))$ defined by $\widetilde{\mathbf{K}}_{(\alpha, \beta)}=\widetilde{\mathbf{K}}_{\alpha}$ and $\widetilde{\mathbf{Q}}_{(\alpha, \beta)}=\widetilde{\mathbf{Q}}_{\alpha}$ respectively. By definition, we have $\left.\mathbf{K}\right|_{K(\{0\})}=\mathbf{K}$ and $\left.\widetilde{\mathbf{Q}}\right|_{K(\{0\})}$ is identified with $\mathbf{Q}$ by Remark 3.8. Then we have a canonical isomorphism $K([0,1)) \times \mathbf{C} \rightarrow \operatorname{Hom}(\widetilde{\mathbf{K}}, \widetilde{\mathbf{Q}})$ such that $\alpha \times 1$ is mapped to the isomorphism sending $U^{*} G\left(\mathbf{s}, e^{\sqrt{-1} \theta}\right) \mathbf{x}$ to $\mathbf{x}$ for $0<b<1$ and sending $U_{\Sigma}^{*} \mathbf{s}$ to $\mathbf{s}$ for $0 \leq b<1 / 2$, which does not depend on the choice of $\mathbf{x}$ or $\mathbf{s}$ representing $[\mathbf{x}]$ or $[\mathbf{s}]$ respectively and is uniquely determined by $\alpha$. Let

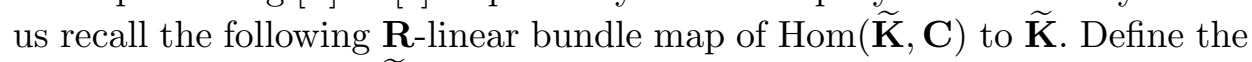
hermitian form $h_{\widetilde{\mathbf{K}}}$ on $\widetilde{\mathbf{K}}$ by $h_{\widetilde{\mathbf{K}}}\left(z_{1} \mathbf{v}, z_{2} \mathbf{v}\right)=z_{1} \bar{z}_{2}\|\mathbf{v}\|^{2}=z_{1} \bar{z}_{2}$, where $\mathbf{v}$ is any vector of length 1 in $\widetilde{\mathbf{K}}_{\alpha}$. Then we have the orientation reversing bundle map over $\mathbf{R}, B_{h}: \widetilde{\mathbf{K}} \rightarrow \operatorname{Hom}(\widetilde{\mathbf{K}}, \mathbf{C})$ defined by $B_{h}(z \mathbf{v})=h_{\widetilde{\mathbf{K}}}(, z \mathbf{v})$, where we note that $h_{\widetilde{\mathbf{K}}}(, z \mathbf{v})$ is a $\mathbf{C}$-homomorphism. Then we have $B_{h}(z \mathbf{v})=\bar{z} B_{h}(\mathbf{v})$. These observations induce the map

$$
\Psi: \operatorname{Hom}(\widetilde{\mathbf{K}} \bigcirc \widetilde{\mathbf{K}}, \widetilde{\mathbf{Q}}) \cong \operatorname{Hom}(\widetilde{\mathbf{K}}, \operatorname{Hom}(\widetilde{\mathbf{K}}, \widetilde{\mathbf{Q}})) \cong \operatorname{Hom}(\widetilde{\mathbf{K}}, \mathbf{C}) \stackrel{B_{h}^{-1}}{\longrightarrow} \widetilde{\mathbf{K}}
$$

For a non-zero vector $\mathbf{x}$ of $\mathbf{C}^{n}$, let $\operatorname{pr}(\mathbf{x})$ denote the orthogonal projection of $\mathbf{C}^{n}$ onto the subspace of dimension 1 generated by $\mathbf{x}$ over $\mathbf{C}$. Since the element $(\alpha, \beta)$ induces the $\operatorname{map} \operatorname{pr}(\mathbf{x}) \circ \beta \mid \widetilde{\mathbf{K}}_{\alpha} \bigcirc \widetilde{\mathbf{K}}_{\alpha}: \widetilde{\mathbf{K}}_{\alpha} \bigcirc \widetilde{\mathbf{K}}_{\alpha} \rightarrow \widetilde{\mathbf{Q}}$, $\Psi$ determines the vector $\Psi\left(\operatorname{pr}(\mathbf{x}) \circ \beta \mid \widetilde{\mathbf{K}}_{\alpha} \bigcirc \widetilde{\mathbf{K}}_{\alpha}\right)$ in $\widetilde{\mathbf{K}}_{\alpha}$. This is written as $u(\alpha, \beta) \mathbf{k}$ by some real number $u(\alpha, \beta) \geq 0$ and some vector $\mathbf{k}$ with length 1 such that $[\mathbf{k}]=\left[U^{*} G\left(\mathbf{s}, e^{\sqrt{-1} \theta}\right) \mathbf{x}\right]$ for $0<b<1$ and $[\mathbf{k}]=\left[U_{\Sigma}^{*} \mathbf{s}\right]$ for $0 \leq b<1 / 2$. We note that $\mathbf{k}$ is determined only when $u(\alpha, \beta)>0$. Let $\mathbf{s}(\alpha, \beta)$ denote $G\left(\mathbf{s}, e^{\sqrt{-1} \theta}\right)^{*} U \mathbf{k}$ for $0<b<1$ and $U_{\Sigma} \mathbf{k}$ for $0 \leq b<1 / 2$. If $u(\alpha, \beta)>0$, then we have that

$$
\operatorname{pr}(\mathbf{s}(\alpha, \beta))(\beta(\mathbf{k}, \mathbf{k}))=u(\alpha, \beta) \mathbf{s}(\alpha, \beta) \quad \text { for } \quad 0 \leq b<1 .
$$


Here set $\bar{u}(\alpha, \beta)=u(\alpha, \beta) /\left(b^{2}+u(\alpha, \beta)^{2}\right)^{1 / 2}$ for $0 \leq b<1$, where $b^{2}+u(\alpha, \beta)^{2}$ never vanishes. Now we set $\mathbf{x}(\alpha, \beta)=\left(1-b^{2}\right)^{1 / 2} \mathbf{s}(\alpha, \beta)$. If $u(\alpha, \beta)=0$, then $\mathbf{x}(\alpha, \beta)$ or $\mathbf{s}(\alpha, \beta)$ represents any vector of length $\left(1-b^{2}\right)^{1 / 2}$ or 1 in $\widetilde{Q}_{\alpha}$ respectively. Furthermore, we set $\mathbf{y}(\alpha, \beta)=u(\alpha, \beta) \mathbf{s}(\alpha, \beta)$, which is always defined. The motivation for this notation is the fact that

$$
\begin{gathered}
\beta\left(\mathbf{x}, e^{\sqrt{-1} \theta}, U\right)\left(U^{*} G\left(\mathbf{s}, e^{\sqrt{-1} \theta}\right) S \mathbf{e}_{n}, U^{*} G\left(\mathbf{s}, e^{\sqrt{-1} \theta}\right) S \mathbf{e}_{n}\right)=\|\mathbf{x}\| \mathbf{s} \\
\text { for } 0<b<1, \\
\beta_{\Sigma}\left(\mathbf{s}, b e^{\sqrt{-1} \theta}, U_{\Sigma}\right)\left(U_{\Sigma}^{*} S \mathbf{e}_{n}, U_{\Sigma}^{*} S \mathbf{e}_{n}\right)=\|\mathbf{x}\| \mathbf{s} \quad \text { for } 0 \leq b<1 / 2 .
\end{gathered}
$$

We note that

(1) The vector $\mathbf{y}(\alpha, \beta)$ is continuous on $\left(\pi_{1}^{2} \mid \Omega^{10}\right)^{-1}(K([0,1))) \cup K(\{1\}) \times$ $\{\mathbf{0}\}$,

(2) If $0<\|\mathbf{x}(\alpha, \beta)\|<1$, equivalently $0<b<1$, then $\|\mathbf{y}(\alpha, \beta)\|=$ $u(\alpha, \beta) /\left(b^{2}+u(\alpha, \beta)^{2}\right)^{1 / 2}<1$,

(3) $u\left(\widetilde{H}\left(\mathbf{x}(\alpha, \beta), e^{\sqrt{-1} \theta}, U\right), \beta\left(\mathbf{x}(\alpha, \beta), e^{\sqrt{-1} \theta}, U\right)\right)=\left(1-b^{2}\right)^{1 / 2}$,

(4) $u\left(\widetilde{H}_{\Sigma}\left(\mathbf{s}(\alpha, \beta), b e^{\sqrt{-1} \theta}, U_{\Sigma}\right), \beta_{\Sigma}\left(\mathbf{s}(\alpha, \beta), b e^{\sqrt{-1} \theta}, U_{\Sigma}\right)\right)=\left(1-b^{2}\right)^{1 / 2}$ and

(5) Consider the case where $b^{2}+u(\alpha, \beta)^{2}=1$, which is, in particular, satisfied for $(\alpha, \beta)$ in $\operatorname{Im}(h)$. Then we have $u(\alpha, \beta)=\bar{u}(\alpha, \beta)$ and $\mathbf{x}(\alpha, \beta)=\mathbf{y}(\alpha, \beta)$.

For an element $(\alpha, \beta)$ of $\left(\pi_{1}^{2} \mid \Omega^{10}\right)^{-1}(K(\{b\}))$ given above, we define $R_{\lambda}(\alpha, \beta)$ to be

$$
\left\{\begin{array}{rr}
\left(\widetilde{H}\left((1-\lambda) \mathbf{x}(\alpha, \beta)+\lambda \mathbf{y}(\alpha, \beta), e^{\sqrt{-1} \theta}, U\right),\right. & \text { for } 0<b<1, \\
\left.(1-\lambda) \beta+\lambda \beta\left(\mathbf{y}(\alpha, \beta), e^{\sqrt{-1} \theta}, U\right)\right) & \text { for } b=1 \text { and } \beta=\mathbf{0}, \\
(\alpha, \mathbf{0}) & \text { for } b=0,
\end{array}\right.
$$

where if $u(\alpha, \beta)=0$, then $\widetilde{H}\left((1-\lambda) \mathbf{x}(\alpha, \beta), e^{\sqrt{-1} \theta}, U\right)$ refers to $\widetilde{H}\left([(1-\lambda) \mathbf{x}(\alpha, \beta)], \mathbf{d}_{a^{\prime} b^{\prime}}, e^{\sqrt{-1} \theta}, U\right)$ with $b^{\prime}=\left(1-(1-\lambda)^{2}\left(1-b^{2}\right)\right)^{1 / 2}$.

Let us see that $R_{\lambda}$ is well defined and continuous. Set $b_{\lambda}(\alpha, \beta)=$ $\left\{1-\|(1-\lambda) \mathbf{x}(\alpha, \beta)+\lambda \mathbf{y}(\alpha, \beta)\|^{2}\right\}^{1 / 2}$. If $0 \leq b_{\lambda}(\alpha, \beta)<1 / 2$ and $0 \leq$ 
$1-\|\mathbf{y}(\alpha, \beta)\|^{2}<1 / 2$, then we may write $R_{\lambda}(\alpha, \beta)$ as a different form $\left(\widetilde{H}_{\Sigma}\left(\mathbf{s}(\alpha, \beta), b_{\lambda}(\alpha, \beta) e^{\sqrt{-1} \theta}, U_{\Sigma}\right),(1-\lambda) \beta+\lambda \beta_{\Sigma}\left(\mathbf{s}(\alpha, \beta),\left(1-\|\mathbf{y}(\alpha, \beta)\|^{2}\right)^{1 / 2} \times\right.\right.$ $\left.e^{\sqrt{-1} \theta}, U_{\Sigma}\right)$ ) by $(3.6),(5.1)$ and (5.2). In particular, if $u(\alpha, \beta)=0$, then $0<$ $b<1, \mathbf{y}(\alpha, \beta)=\mathbf{0}$ and $\beta\left(\mathbf{y}(\alpha, \beta), e^{\sqrt{-1} \theta}, U=\mathbf{0}\right.$. If $b=0$, then $u(\alpha, \beta)>0$ and $(1-\lambda) \mathbf{x}(\alpha, \beta)+\lambda \mathbf{y}(\alpha, \beta)=(1-\lambda) \mathbf{x}(\alpha, \beta)+\lambda \mathbf{x}(\alpha, \beta)=\mathbf{x}(\alpha, \beta)=\mathbf{s}(\alpha, \beta)$. Therefore, $R_{\lambda}$ is well defined and continuous.

We see that $R_{\lambda}$ maps $\left(\pi_{1}^{2} \mid \Omega^{10}\right)^{-1}(K((0,1)))$ into $\left(\pi_{1}^{2} \mid \Omega^{10}\right)^{-1}(K((0,1)))$ $\cup K(\{1\}) \times\{\mathbf{0}\}$. If $0<\|\mathbf{x}(\alpha, \beta)\|<1$, or equivalently $0<b<1$, then $\|(1-\lambda) \mathbf{x}(\alpha, \beta)+\lambda \mathbf{y}(\alpha, \beta)\|$ is less than 1 and is equal to 0 only when $\lambda=1$ and $u(\alpha, \beta)=0$. Furthermore, if $\lambda=1, \bar{u}(\alpha, \beta)=0$ and $0<\|\mathbf{x}(\alpha, \beta)\|<1$, then $R_{1}(\alpha, \beta)=\left(\widetilde{H}\left(\mathbf{0}, e^{\sqrt{-1} \theta}, U\right), \mathbf{0}\right)$, since $\beta\left(\mathbf{0}, e^{\sqrt{-1} \theta}, U\right)=\mathbf{0}$.

We see that $R_{\lambda}$ maps $\left(\pi_{1}^{2} \mid \Omega^{10}\right)^{-1}(K(\{0\}))$ into $\left(\pi_{1}^{2} \mid \Omega^{10}\right)^{-1}(K(\{0\}))$. By definition, we have that $\operatorname{pr}(\mathbf{s}(\alpha, \beta)))\left((1-\lambda) \beta+\lambda \beta_{\Sigma}\left(\mathbf{s}(\alpha, \beta), \mathbf{0}, U_{\Sigma}\right)\right)$ ( $\left.U_{\Sigma}^{*} \mathbf{s}(\alpha, \beta), U_{\Sigma}^{*} \mathbf{s}(\alpha, \beta)\right)=((1-\lambda) u(\alpha, \beta)+\lambda) \mathbf{s}(\alpha, \beta)$. Since $(\alpha, \beta) \in \Sigma^{10}$, we have $u(\alpha, \beta)>0$ and so $(1-\lambda) u(\alpha, \beta)+\lambda>0$.

By definition, the image of $R_{1}$ is contained in $\operatorname{Im}(h)$. It is easy to see that $R_{0}=\mathrm{id}$. It follows from (3), (4) and (5) that $R_{\lambda} \mid \operatorname{Im}(h)$ is constantly equal to $\operatorname{id}_{\operatorname{Im}(h)}$.

\section{$\S 6 . \quad \mathrm{SU}(n) \times \mathrm{SU}(n)$ action}

In this section the unit vector $\mathbf{e}_{n+1}^{\prime}$ of $\mathbf{C}^{n+1}$ in Section 4 is written as $\mathbf{e}_{n+1}$ to avoid confusion. We consider the following action of $\mathrm{SU}(n) \times \mathrm{SU}(n)$ on $J^{2}(n, n)$. An element $\left(O^{\prime}, O^{*}\right)$ of $\mathrm{SU}(n) \times \mathrm{SU}(n)$ acts on each element $(\alpha, \beta)$ of $J^{2}(n, n)$ by

$$
\left(\left(O^{\prime}, O^{*}\right) \cdot(\alpha, \beta)\right)(\mathbf{a}, \mathbf{b}, \mathbf{c})=\left(O^{\prime} \alpha(O \mathbf{a}), O^{\prime} \beta(O \mathbf{b}, O \mathbf{c})\right)
$$

and also acts on each element $M$ of $\mathrm{SU}(n+1)$ by

$$
\left(O^{\prime}, O^{*}\right) \cdot M=\left(O^{\prime} \dot{+}(1)\right) M(O \dot{+}(1))
$$

Note that $\Omega^{10}$ is invariant with respect to this action. We will prove that $i_{n}$ is equivariant with respect to these actions of $\mathrm{SU}(n) \times \mathrm{SU}(n)$. Its proof needs a complicated observation about the embedding $i_{n}$. First we prepare two lemmas.

LEMMA 6.1. Let $M \mathbf{e}_{n+1}$ be written as $\mathbf{z}={ }^{t}\left(x_{1}, \ldots, x_{n}, z_{n+1}\right)$ with $z_{n+1}=b e^{\sqrt{-1} \theta}$ as above. Let $\mathbf{w}$ be $\left(O^{\prime} \dot{+}(1)\right) \mathbf{z}$ for an element $O^{\prime}$ of $\mathrm{SU}(n)$. Then we have 
(1) $r(\mathbf{w})^{-1}\left(O^{\prime} \dot{+}(1)\right)=\left(O^{\prime} \dot{+}(1)\right) r(\mathbf{z})^{-1} \quad$ for $0<b \leq 1$,

(2) $r_{\Sigma}(\mathbf{w})^{-1}\left(O^{\prime} \dot{+}(1)\right)=\left(O^{\prime} \dot{+}(1)\right) r_{\Sigma}(\mathbf{z})^{-1} \quad$ for $0 \leq b<1 / 2$,

Proof. (1) It is enough to prove $\left(O^{\prime} \dot{+}(1)\right) r(\mathbf{z})\left(O^{\prime} \dot{+}(1)\right)^{*}=r(\mathbf{w})$. By the property (4.1) of $r(\mathbf{w})$ we have

$$
\begin{aligned}
& r(\mathbf{w})\left(\mathbf{e}_{n+1}\right)=e^{-\sqrt{-1} \theta} \mathbf{w}=e^{-\sqrt{-1} \theta}\left(O^{\prime}+(1)\right) \mathbf{z}, \\
& r(\mathbf{w})\left(\mathbf{w}-b e^{\sqrt{-1} \theta} \mathbf{e}_{n+1}\right)=b \mathbf{w}-e^{\sqrt{-1} \theta} \mathbf{e}_{n+1} \\
& =b\left(O^{\prime}+(1)\right) \mathbf{z}-e^{\sqrt{-1} \theta} \mathbf{e}_{n+1} \quad \text { and } \\
& r(\mathbf{w}) \mathbf{f}=\mathbf{f} \text { if } \mathbf{f} \text { is orthogonal to } \mathbf{e}_{n+1} \text { and } \mathbf{w}-b e^{\sqrt{-1} \theta} \mathbf{e}_{n+1} \text {. }
\end{aligned}
$$

On the other hand, we have

$$
\begin{aligned}
\left(O^{\prime} \dot{+}(1)\right) r(\mathbf{z}) & \left(O^{\prime} \dot{+}(1)\right)^{*}\left(\mathbf{e}_{n+1}\right) \\
= & \left(O^{\prime} \dot{+}(1)\right) r(\mathbf{z})\left(\mathbf{e}_{n+1}\right) \\
= & \left(O^{\prime} \dot{+}(1)\right) e^{-\sqrt{-1} \theta} \mathbf{z} \\
= & e^{-\sqrt{-1} \theta}\left(O^{\prime} \dot{+}(1)\right) \mathbf{z}, \\
\left(O^{\prime} \dot{+}(1)\right) r(\mathbf{z}) & \left(O^{\prime} \dot{+}(1)\right)^{*}\left(\mathbf{w}-b e^{\sqrt{-1} \theta} \mathbf{e}_{n+1}\right) \\
= & \left(O^{\prime} \dot{+}(1)\right) r(\mathbf{z})\left(O^{\prime} \dot{+}(1)\right)^{*}\left(O^{\prime} \dot{+}(1)\right)\left(\mathbf{z}-z_{n+1} \mathbf{e}_{n+1}\right) \\
= & \left(O^{\prime} \dot{+}(1)\right) r(\mathbf{z})\left(\mathbf{z}-z_{n+1} \mathbf{e}_{n+1}\right) \\
= & \left(O^{\prime} \dot{+}(1)\right)\left(b \mathbf{z}-e^{\sqrt{-1} \theta} \mathbf{e}_{n+1}\right) \\
= & b\left(O^{\prime} \dot{+}(1)\right) \mathbf{z}-e^{\sqrt{-1} \theta} \mathbf{e}_{n+1} .
\end{aligned}
$$

Since $\mathbf{f}$ satisfies $\left(\mathbf{f}, \mathbf{e}_{n+1}\right)=\left(\mathbf{f},\left(O^{\prime} \dot{+}(1)\right)\left(\mathbf{z}-z_{n+1} \mathbf{e}_{n+1}\right)\right)=0$, we have $\left(\left(O^{\prime} \dot{+}(1)\right)^{*} \mathbf{f}, \mathbf{e}_{n+1}\right)=\left(\left(O^{\prime} \dot{+}(1)\right)^{*} \mathbf{f}, \mathbf{z}-z_{n+1} \mathbf{e}_{n+1}\right)=0$. It follows from the property (4.1-(iii)) of $r(\mathbf{z})$ that

$$
\left(O^{\prime} \dot{+}(1)\right) r(\mathbf{z})\left(O^{\prime} \dot{+}(1)\right)^{*} \mathbf{f}=\left(O^{\prime} \dot{+}(1)\right)\left(O^{\prime} \dot{+}(1)\right)^{*} \mathbf{f}=\mathbf{f} .
$$

Thus we obtain

$$
r(\mathbf{w})=\left(O^{\prime} \dot{+}(1)\right) r(\mathbf{z})\left(O^{\prime} \dot{+}(1)\right)^{*}
$$

(2) The proof is similar. By definition we have 


$$
\begin{aligned}
r_{\Sigma}(\mathbf{w})\left(\mathbf{e}_{n+1}\right)=\mathbf{w}=\left(O^{\prime}\right. & \dot{+}(1)) \mathbf{z}, \\
r_{\Sigma}(\mathbf{w})\left(\mathbf{w}-b e^{\sqrt{-1} \theta} \mathbf{e}_{n+1}\right) & =b e^{\sqrt{-1} \theta} \mathbf{w}-\mathbf{e}_{n+1} \\
& =b e^{-\sqrt{-1} \theta}\left(O^{\prime} \dot{+}(1)\right) \mathbf{z}-\mathbf{e}_{n+1} \quad \text { and }
\end{aligned}
$$

$r_{\Sigma}(\mathbf{w}) \mathbf{f}=\mathbf{f}$ if $\mathbf{f}$ is orthogonal to $\mathbf{e}_{n+1}$ and $\mathbf{w}-b e^{\sqrt{-1} \theta} \mathbf{e}_{n+1}$.

On the other hand, we have

$$
\begin{aligned}
&\left(O^{\prime} \dot{+}(1)\right) r_{\Sigma}(\mathbf{z})\left(O^{\prime} \dot{+}(1)\right)^{*}\left(\mathbf{e}_{n+1}\right) \\
&=\left(O^{\prime} \dot{+}(1)\right) r_{\Sigma}(\mathbf{z})\left(\mathbf{e}_{n+1}\right) \\
&=\left(O^{\prime} \dot{+}(1)\right)(\mathbf{z}), \\
&\left(O^{\prime} \dot{+}(1)\right) r_{\Sigma}(\mathbf{z})\left(O^{\prime} \dot{+}(1)\right)^{*}\left(O^{\prime} \dot{+}(1)\right)\left(\mathbf{z}-z_{n+1} \mathbf{e}_{n+1}\right) \\
&=\left(O^{\prime} \dot{+}(1)\right) r_{\Sigma}(\mathbf{z})\left(\mathbf{z}-z_{n+1} \mathbf{e}_{n+1}\right) \\
&=\left(O^{\prime} \dot{+}(1)\right)\left(\bar{z}_{n+1} \mathbf{z}-\mathbf{e}_{n+1}\right) \\
&=\bar{z}_{n+1}\left(O^{\prime} \dot{+}(1)\right) \mathbf{z}-\mathbf{e}_{n+1} .
\end{aligned}
$$

Similarly we have that $\left(\left(O^{\prime} \dot{+}(1)\right)^{*} \mathbf{f}, \mathbf{e}_{n+1}\right)=\left(\left(O^{\prime} \dot{+}(1)\right)^{*} \mathbf{f}, \mathbf{z}-z_{n+1} \mathbf{e}_{n+1}\right)=$ 0 . It follows from the property $(4.2-(\mathrm{iii}))$ of $r_{\Sigma}(\mathbf{z})$ that

$$
\left(O^{\prime} \dot{+}(1)\right) r_{\Sigma}(\mathbf{z})\left(O^{\prime} \dot{+}(1)\right)^{*} \mathbf{f}=\left(O^{\prime} \dot{+}(1)\right)\left(O^{\prime} \dot{+}(1)\right)^{*} \mathbf{f}=\mathbf{f}
$$

Thus we obtain

$$
r_{\Sigma}(\mathbf{w})=\left(O^{\prime} \dot{+}(1)\right) r_{\Sigma}(\mathbf{z})\left(O^{\prime} \dot{+}(1)\right)^{*}
$$

Lemma 6.2. Set $M^{\prime}=\left(O^{\prime} \dot{+}(1)\right) M(O+(1))$ for $O$ and $O^{\prime}$ in $\mathrm{SU}(n)$. Then we have
(1) $U\left(M^{\prime}\right)=I_{\theta} O^{\prime} I_{-\theta} U(M) O$
for $0<b \leq 1$,
(2) $U_{\Sigma}\left(M^{\prime}\right)=O^{\prime} U_{\Sigma}(M) O$
for $0 \leq b<1 / 2$.

Proof. It follows from Lemma 6.1 that

$$
\text { (1) } \quad r(\mathbf{w})^{-1} M^{\prime}=r(\mathbf{w})^{-1}\left(O^{\prime} \dot{+}(1)\right) M(O \dot{+}(1))
$$




$$
\begin{aligned}
& =\left(O^{\prime} \dot{+}(1)\right) r(\mathbf{z})^{-1} M(O \dot{+}(1)) \\
& =\left(O^{\prime} \dot{+}(1)\right)\left(I_{-\theta} U(M) \dot{+}\left(e^{\sqrt{-1} \theta}\right)\right)(O \dot{+}(1)) \\
& =O^{\prime} I_{-\theta} U(M) O \dot{+}\left(e^{\sqrt{-1} \theta}\right) \\
& =I_{-\theta}\left(I_{\theta} O^{\prime} I_{-\theta} U(M) O\right) \dot{+}\left(e^{\sqrt{-1} \theta}\right) \\
r_{\Sigma}(\mathbf{w})^{-1} M^{\prime} & =r_{\Sigma}(\mathbf{w})^{-1}\left(O^{\prime} \dot{+}(1)\right) M(O \dot{+}(1)) \\
& =\left(O^{\prime} \dot{+}(1)\right) r_{\Sigma}(\mathbf{z})^{-1} M(O \dot{+}(1)) \\
& =\left(O^{\prime} \dot{+}(1)\right)\left(U_{\Sigma}(M) \dot{+}(1)\right)(O \dot{+}(1)) \\
& =O^{\prime} U_{\Sigma}(M) O \dot{+}(1)
\end{aligned}
$$

Thus (1) and (2) follow from the definitions of $U\left(M^{\prime}\right)$ and $U_{\Sigma}\left(M^{\prime}\right)$ respectively.

We are ready to prove the following.

Proposition 6.3. The embedding $i_{n}$ is equivariant with respect to the actions of $\mathrm{SU}(n) \times \mathrm{SU}(n)$ on $\mathrm{SU}(n+1)$ and $J^{2}(n, n)$.

Proof. We use the notations given in the definition of $i_{n}$ and let $M$, $O^{\prime}, O$ and $M^{\prime}$ with $\mathbf{w}=M^{\prime} \mathbf{e}_{n+1}$ and $\mathbf{z}=M \mathbf{e}_{n+1}$ be as above. We have that if $b<1$, then $\mathbf{s}\left(M^{\prime}\right)=O^{\prime} \mathbf{s}(M)$. Then we obtain the following.

If $0<b<1$, then

$$
\begin{aligned}
\widetilde{H}\left(\mathbf{x}\left(M^{\prime}\right), e^{\sqrt{-1} \theta}, U\left(M^{\prime}\right)\right) & =O^{\prime} S \Delta\left(\mathbf{d}_{a b}\right) S^{*} O^{\prime *} I_{-\theta} U\left(M^{\prime}\right) \\
& =O^{\prime} S \Delta\left(\mathbf{d}_{a b}\right) S^{*} O^{\prime *} I_{-\theta} I_{\theta} O^{\prime} I_{-\theta} U(M) O \\
& =O^{\prime} S \Delta\left(\mathbf{d}_{a b}\right) S^{*} I_{-\theta} U(M) O \\
& =O^{\prime} \widetilde{H}\left(\mathbf{x}(M), e^{\sqrt{-1} \theta}, U(M)\right) O .
\end{aligned}
$$

If $b=1$, then

$$
\begin{aligned}
\widetilde{H}\left(\mathbf{0}, e^{\sqrt{-1} \theta}, U\left(M^{\prime}\right)\right) & =(1 / \sqrt{n}) I_{-\theta} U\left(M^{\prime}\right) \\
& =O^{\prime}(1 / \sqrt{n}) I_{-\theta} U(M) O \\
& =O^{\prime} \widetilde{H}\left(\mathbf{0}, e^{\sqrt{-1} \theta}, U(M)\right) O .
\end{aligned}
$$


Let $0<b<1$. Since

$$
\begin{aligned}
G\left(\mathbf{s}\left(M^{\prime}\right), e^{\sqrt{-1} \theta}\right)^{*} U\left(M^{\prime}\right) & =O^{\prime} S I_{\theta} S^{*} O^{*} I_{-\theta} I_{\theta} O^{\prime} I_{-\theta} U(M) O \\
& =O^{\prime} S I_{\theta} S^{*} I_{-\theta} U(M) O
\end{aligned}
$$

we have

$$
\begin{aligned}
& \beta\left(\mathbf{x}\left(M^{\prime}\right), e^{\sqrt{-1} \theta}, U\left(M^{\prime}\right)\right)(\mathbf{a}, \mathbf{b}) \\
= & \left\{{ }^{t} \mathbf{a}^{t}\left(G\left(\mathbf{s}\left(M^{\prime}\right), e^{\sqrt{-1} \theta}\right)^{*} U\left(M^{\prime}\right)\right) \bar{O}^{\prime} \bar{S}\right. \\
& \times \Delta\left(0, \ldots, 0,\left\|\mathbf{x}\left(M^{\prime}\right)\right\| S^{*} O^{\prime *} G\left(\mathbf{s}\left(M^{\prime}\right), e^{\sqrt{-1} \theta}\right)^{*} U\left(M^{\prime}\right) \mathbf{b}\right\} \mathbf{s}\left(M^{\prime}\right) \\
= & \left\{{ }^{t} \mathbf{a}^{t} O^{t} U(M) I_{-\theta} \bar{S} I_{\theta}{ }^{t} S \bar{S}\right. \\
& \left.\quad \times \Delta(0, \ldots, 0,\|\mathbf{x}(M)\|) S^{*} S I_{\theta} S^{*} I_{-\theta} U(M) O \mathbf{b}\right\} O^{\prime} \mathbf{s}(M) \\
= & \left\{{ }^{t}(O \mathbf{a})^{t}\left(G\left(\mathbf{s}(M), e^{\sqrt{-1} \theta}\right)^{*} U(M)\right) \bar{S}\right. \\
\quad & \left.\times \Delta(0, \ldots, 0,\|\mathbf{x}(M)\|) S^{*} G\left(\mathbf{s}(M), e^{\sqrt{-1} \theta}\right)^{*} U(M) O \mathbf{b}\right\} O^{\prime} \mathbf{s}(M) \\
= & O^{\prime} \beta\left(\mathbf{x}(M), e^{\sqrt{-1} \theta}, U(M)\right)(O \mathbf{a}, O \mathbf{b}) .
\end{aligned}
$$

This equality also holds in the case of $b=1$.

If $0 \leq b<1 / 2$, then

$$
\begin{aligned}
\widetilde{H}_{\Sigma}\left(\mathbf{s}\left(M^{\prime}\right), b e^{\sqrt{-1} \theta}, U_{\Sigma}\left(M^{\prime}\right)\right) & =O^{\prime} S \Delta\left(\mathbf{d}_{a b}\right) I_{-\theta} S^{*} O^{\prime *} U_{\Sigma}\left(M^{\prime}\right) \\
& =O^{\prime} S \Delta\left(\mathbf{d}_{a b}\right) I_{-\theta} S^{*} O^{\prime *} O^{\prime} U_{\Sigma}(M) O \\
& =O^{\prime} S \Delta\left(\mathbf{d}_{a b}\right) I_{-\theta} S^{*} U_{\Sigma}(M) O \\
& =O^{\prime} \widetilde{H}_{\Sigma}\left(\mathbf{s}(M), b e^{\sqrt{-1} \theta}, U_{\Sigma}(M)\right) O
\end{aligned}
$$

and

$$
\begin{aligned}
\beta_{\Sigma} & \left(\mathbf{s}\left(M^{\prime}\right), b e^{\sqrt{-1} \theta}, U_{\Sigma}\left(M^{\prime}\right)\right)(\mathbf{a}, \mathbf{b}) \\
& =\left\{{ }^{t} \mathbf{a}^{t} U_{\Sigma}\left(M^{\prime}\right) \bar{O}^{\prime} \bar{S} \Delta\left(0, \ldots, 0,\left\|\mathbf{x}\left(M^{\prime}\right)\right\|\right) S^{*} O^{\prime *} U_{\Sigma}\left(M^{\prime}\right) \mathbf{b}\right\} \mathbf{s}\left(M^{\prime}\right) \\
& =\left\{{ }^{t} \mathbf{a}^{t} O^{t} U_{\Sigma}(M)^{t} O^{\prime} \bar{O}^{\prime} \bar{S} \Delta(0, \ldots, 0,\|\mathbf{x}(M)\|) S^{*} O^{\prime *} O^{\prime} U_{\Sigma}(M) O \mathbf{b}\right\} O^{\prime} \mathbf{s}(M) \\
& =\left\{{ }^{t}(O \mathbf{a})^{t} U_{\Sigma}(M) \bar{S} \Delta(0, \ldots, 0,\|\mathbf{x}(M)\|) S^{*} U_{\Sigma}(M) O \mathbf{b}\right\} O^{\prime} \mathbf{s}(M) \\
& =O^{\prime} \beta_{\Sigma}\left(\mathbf{s}(M), b e^{\sqrt{-1} \theta}, U_{\Sigma}(M)\right)(O \mathbf{a}, O \mathbf{b}) .
\end{aligned}
$$

This proves that $i_{n}$ is equivariant with respect to the actions of $\mathrm{SU}(n) \times$ $\mathrm{SU}(n)$.

Proof of Theorem 1(2). The assertion follows from Theorem 5.5 and Proposition 6.3. 


\section{$\S 7$. Holomorphic fold maps}

Let $J^{2}(N, P)$ be the complex 2-jet space of complex manifolds $N$ and $P$. Let $\pi_{N}$ and $\pi_{P}$ be the projections mapping a jet to its source and target respectively. Let $L^{2}(n)$ be the group of 2-jets of all biholomorphic map germs $\left(\mathbf{C}^{n}, 0\right) \rightarrow\left(\mathbf{C}^{n}, 0\right)$. The map $\pi_{N} \times \pi_{P}: J^{2}(N, P) \rightarrow N \times P$ gives the structure of a fibre bundle with fibre $J^{2}(n, n)$ having the structure group $L^{2}(n) \times L^{2}(n)$. Let $\operatorname{Hom}(T N \oplus(T N \bigcirc T N), T P)$ be the vector bundle over $N \times P$ with structure group $G L(n ; \mathbf{C}) \times G L(n ; \mathbf{C})$, which is the union of all spaces $\operatorname{Hom}\left(T_{x} N \oplus\left(T_{x} N \bigcirc T_{x} N\right), T_{y} P\right)$ for $(x, y)$ of $N \times P$, where $T_{x} N \bigcirc T_{x} N$ denotes the 2-fold symmetric product of $T_{x} N$. If a basis of $\mathbf{C}^{n}$ is fixed, then we have the canonical C-linear isomorphism $j: J^{2}(n, n) \rightarrow$ $\operatorname{Hom}\left(\mathbf{C}^{n} \oplus\left(\mathbf{C}^{n} \bigcirc \mathbf{C}^{n}\right), \mathbf{C}^{n}\right)$ by considering Taylor expansions. It is clear that $j$ is equivariant with respect to the actions of $G L(n ; \mathbf{C}) \times G L(n ; \mathbf{C})$ on both spaces $J^{2}(n, n)$ and $\operatorname{Hom}\left(\mathbf{C}^{n} \oplus\left(\mathbf{C}^{n} \bigcirc \mathbf{C}^{n}\right), \mathbf{C}^{n}\right)$. Since $G L(n ; \mathbf{C})$ is naturally a subgroup of $L^{2}(n)$ and the quotient space $L^{2}(n) / G L(n ; \mathbf{C})$ is contractible, the structure group $L^{2}(n) \times L^{2}(n)$ of the fibre bundle $\pi_{N} \times \pi_{P}$ : $J^{2}(N, P) \rightarrow N \times P$ is reduced to $G L(n ; \mathbf{C}) \times G L(n ; \mathbf{C})$. Hence it follows from [St, 12.6 Corollary] that we obtain a bundle map

$$
J: J^{2}(N, P) \longrightarrow \operatorname{Hom}(T N \oplus(T N \bigcirc T N), T P)
$$

which is uniquely determined up to homotopy.

Let $z=j_{x}^{2} f$ with $y=f(x)$ be a 2-jet in $J_{x, y}^{2}(N, P)$, which is the subset of $J^{2}(N, P)$ consisting of all 2-jets of germs of $(N, x)$ to $(P, y)$. Set $\mathbf{D}=\pi_{N}^{*}(T N)$ and $\mathbf{P}=\pi_{P}^{*}(T P)$. Then there is a homomorphism $d_{1}: \mathbf{D} \rightarrow$ $\mathbf{P}$ defined as follows. Let $\mathbf{D}_{z}$ and $\mathbf{P}_{z}$ be the fibres of $\mathbf{D}$ and $\mathbf{P}$ over $z$ respectively. Then $d_{1, z}: \mathbf{D}_{z} \rightarrow \mathbf{P}_{z}$ refers to $d f: T_{x} N \rightarrow T_{y} P$. We define $\Sigma^{i}(N, P)$ to be the set of all jets $z$ with $\operatorname{dim}\left(\operatorname{Ker}\left(d_{1 . z}\right)\right)=i$. Then we have the subbundle $\mathbf{K}=\operatorname{Ker}\left(d_{1}\right)$ and the cokernel bundle $\mathbf{Q}=\operatorname{Cok}\left(d_{1}\right)$ over $\Sigma^{i}(N, P)$. In [Bo, p.50, Lemma 7.13 and Theorem 7.14] (see also [Ma, §2]) the second intrinsic derivative $d_{2}: \mathbf{K} \rightarrow \operatorname{Hom}(\mathbf{K}, \mathbf{Q})$ has been defined by using the second derivative of $z$. We define $\Sigma^{10}(N, P)$ to be the set of all jets $z$ such that $\operatorname{dim}\left(\operatorname{Ker}\left(d_{1, z}\right)\right)=1$ and $d_{2, z}: \mathbf{K}_{z} \rightarrow \operatorname{Hom}\left(\mathbf{K}_{z}, \mathbf{Q}_{z}\right)$ is an isomorphism. Let $\Omega^{10}(N, P)$ be the union of the set of all regular jets and $\Sigma^{10}(N, P)$.

There is a canonical identification of $J^{k}(n, n)$ with $J_{0,0}^{k}\left(\mathbf{C}^{n}, \mathbf{C}^{n}\right)$. In $\operatorname{Hom}(T N \oplus(T N \bigcirc T N), T P)$ we can also define $\Sigma^{1}(N, P)^{\prime}, \Sigma^{10}(N, P)^{\prime}$ and $\Omega^{10}(N, P)^{\prime}$ associated with $\Sigma^{1}, \Sigma^{10}$ and $\Omega^{10}$ in Section 1 respectively. The 
two constructions above associated with $\Sigma^{1}, \Sigma^{10}$ and $\Omega^{10}$ correspond with each other by $J$. Then $\Omega^{10}(N, P)$ and $\Omega^{10}(N . P)^{\prime}$ are the subbundles of $J^{2}(N, P)$ and $\operatorname{Hom}(T N \oplus(T N \bigcirc T N), T P)$ respectively. Then $J$ induces a bundle map of $\Omega^{10}(N, P)$ to $\Omega^{10}(N, P)^{\prime}$.

For an $n$-dimensional complex manifold $M$, let us recall that an $\mathrm{SU}(n)$ structure of $T M$ is a reduction $(E, \varphi)$ of the structure group $G L(n ; \mathbf{C})$ to $\mathrm{SU}(n)$, where $E$ is an $n$-dimensional $\mathrm{SU}(n)$-vector bundle over $M$ and $\varphi$ : $T M \rightarrow E$ is a bundle map covering $\operatorname{id}_{M}$ (see [St, 9.2]). Two SU( $n$ )-structures $\left(E_{1}, \varphi_{1}\right)$ and $\left(E_{2}, \varphi_{2}\right)$ of $T M$ are equivalent if there exists an $\mathrm{SU}(n)$-bundle $\operatorname{map} B: E_{1} \rightarrow E_{2}$ such that $\varphi_{2}=B \circ \varphi_{1}$. Consider the spherical fibre space $p^{\prime}: \operatorname{BSU}(n) \rightarrow \mathrm{BU}(n)$ with fibre $S^{1}$ induced from the inclusion of $\operatorname{SU}(n)$ into $\mathrm{U}(n)$. Let $c_{T M}: M \rightarrow \mathrm{BU}(n)$ be the classifying map of $T M$. It is well known that equivalence classes of $\mathrm{SU}(n)$-structures of $T M$ correspond bijectively to homotopy classes of continuous maps $c: M \rightarrow \mathrm{BSU}(n)$ with $p^{\prime} \circ c=c_{T M}$.

Suppose that $\operatorname{SU}(n)$-structures $\left(E, \varphi_{N}\right)$ and $\left(F, \varphi_{P}\right)$ of $T N$ and $T P$ are given respectively. Then we can define the canonical bundle map

$$
\Phi: \operatorname{Hom}(T N \oplus(T N \bigcirc T N), T P) \longrightarrow \operatorname{Hom}(E \oplus(E \bigcirc E), F)
$$

by using $\varphi_{N}$ and $\varphi_{P}$. The map $\Phi \circ J$ induces a biholomorphic map between fibres $J_{x, y}^{2}(N, P)$ and $\operatorname{Hom}\left(E_{x} \oplus\left(E_{x} \bigcirc E_{x}\right), F_{y}\right)$ (however, $\Phi$ may not be biholomorphic in general). On the other hand, we have the subbundle $\mathrm{SU}\left(E \oplus \theta_{N}, F \oplus \theta_{P}\right)$ of $\operatorname{Hom}\left(E \oplus \theta_{N}, F \oplus \theta_{P}\right)$ associated with $\mathrm{SU}(n+1)$.

We shall apply the embedding $i_{n}: \mathrm{SU}(n+1) \rightarrow \Omega^{10}\left(\subset \operatorname{Hom}\left(\mathbf{C}^{n} \oplus\right.\right.$ $\left.\left.\left(\mathbf{C}^{n} \bigcirc \mathbf{C}^{n}\right), \mathbf{C}^{n}\right)\right)$ to $\mathrm{SU}\left(E \oplus \theta_{N}, F \oplus \theta_{P}\right)$ and $\operatorname{Hom}(E \oplus(E \bigcirc E), F)$. Let $i(N, P)^{\prime}$ be the map of $\mathrm{SU}\left(E \oplus \theta_{N}, F \oplus \theta_{P}\right)$ to $\Phi\left(\Omega^{10}(N, P)^{\prime}\right)$ associated with $i_{n}$. Then we obtain a subspace homeomorphic to $\mathrm{SU}(n+1)$ denoted by $\mathrm{SU}_{x, y}(E, F)$ in $\operatorname{Hom}\left(E_{x} \oplus\left(E_{x} \bigcirc E_{x}\right), F_{y}\right)$. This space is well defined by Proposition 6.3. The space $\mathrm{SU}(E, F)$ is defined to be the union of all spaces $\mathrm{SU}_{x, y}(E, F)$ in $\Phi\left(\Omega^{10}(N, P)^{\prime}\right)$, where $(x, y)$ varies all over $N \times P$. It becomes a subbundle with structure group $\mathrm{SU}(n) \times \mathrm{SU}(n)$ coming from those of $E$ and $F$. It is clear that the image of $i(N, P)^{\prime}$ coincides with $\mathrm{SU}(E, F)$ and is homotopy equivalent to $\Phi\left(\Omega^{10}(N, P)^{\prime}\right)$ by Theorem $1(2)$. Now we define the map $i(N, P)$ to be

$$
\left(\Phi \circ J \mid \Omega^{10}(N, P)\right)^{-1} \circ i(N, P)^{\prime}: \mathrm{SU}\left(E \oplus \theta_{N}, F \oplus \theta_{P}\right) \longrightarrow \Omega^{10}(N, P)
$$


Proof of Theorem 2. (1) The map $i(N, P)$ gives a homotopy equivalence of fibre bundles, since $\Phi \circ J \mid \Omega^{10}(N, P)$ is a bundle map and $i(N, P)^{\prime}$ is a fibre homotopy equivalence.

(2) Let $i(N, P)^{-1}: \Omega^{10}(N, P) \rightarrow \mathrm{SU}\left(E \oplus \theta_{N}, F \oplus \theta_{P}\right)$ be the homotopy inverse of $i(N, P)$. For a holomorphic fold map $f$, the section $j^{2} f$ determines the homotopy class of a section $i(N, P)^{-1} \circ j^{2} f$ of $\mathrm{SU}\left(E \oplus \theta_{N}, F \oplus \theta_{P}\right)$. This gives the homotopy class of an $\mathrm{SU}(n+1)$-bundle map $\tilde{f}: E \oplus \theta_{N} \rightarrow F \oplus \theta_{P}$ covering $f$ in Theorem $2(2)$.

Proof of Corollary 3. Since the first Chern classes of $N$ and $P$ vanish, there exist $\mathrm{SU}(n)$-structures $\left(E, \varphi_{N}\right)$ and $\left(F, \varphi_{P}\right)$ of $T N$ and $T P$ respectively. Consider the spherical fibre space $p: \operatorname{BSU}(n) \rightarrow \operatorname{BSU}(n+1)$ with fibre $S^{2 n+1}$ induced from the inclusion of $\mathrm{SU}(n)$ into $\mathrm{SU}(n+1)$. Let $c_{N}: N \rightarrow \operatorname{BSU}(n)$ and $c_{P}: P \rightarrow \operatorname{BSU}(n)$ denote the classifying maps of $E$ and $F$ respectively. Then $p \circ c_{N}$ and $p \circ c_{P} \circ f$ are the classifying maps of $T N \oplus \theta_{N}^{1}$ and $f^{*}(T P) \oplus \theta_{N}^{1}$ respectively. By Theorem $2(2)$, there is a homotopy $c: N \times I \rightarrow \operatorname{BSU}(n+1)$ between $p \circ c_{N}$ and $p \circ c_{P} \circ f$. Let $c^{*}(p): c^{*}(\operatorname{BSU}(n)) \rightarrow N \times I$ be the induced fibre space. By applying the obstruction theorem $([\mathrm{St}])$, the obstructions to extending the induced sections $c^{*}\left(c_{N}\right)$ and $c^{*}\left(c_{P} \circ f\right)$ to a section defined on $N \times I$ lie in $H^{i}\left(N \times I, N \times\{0,1\} ; \pi_{i-1}\left(S^{2 n+1}\right)\right)(i=0, \ldots, 2 n+1)$, which vanish for all $i$. Hence, there exists a section $c^{\prime}: N \times I \rightarrow c^{*}(\operatorname{BSU}(n))$ with $c^{\prime} \mid N \times 0=c^{*}\left(c_{N}\right)$ and $c^{\prime} \mid N \times 1=c^{*}\left(c_{P} \circ f\right)$. This implies that there exists an $\mathrm{SU}(n)$-bundle map of $E$ to $f^{*}(F)$, which yields an $\mathrm{SU}(n)$-bundle map $B: E \rightarrow F$. Thus we obtain a bundle map $\varphi_{P}^{-1} \circ B \circ \varphi_{N}: T N \rightarrow T P$ covering $f$.

Remark 7.1. Theorem 2 does not hold for general complex manifolds. The holomorphic fold map $f: \mathbf{C P}^{1} \rightarrow \mathbf{C P}^{1}$ defined by $f([z])=\left[z^{2}\right]$ has the property that $f^{*}\left(C_{1}\left(\mathbf{C P}^{1}\right)\right)=2 C_{1}\left(\mathbf{C P}^{1}\right)$. Hence $T\left(\mathbf{C P}^{1}\right)$ is not even stably equivalent to $f^{*}\left(T\left(\mathbf{C P}^{1}\right)\right)$.

EXAMPLE 7.2. (1) We consider the following Hopf manifolds (cf. [K, Example 2.9]). Let $G$ be the infinite cyclic group generated by the automorphism $g$ of $\mathbf{C}^{n} \backslash\{\mathbf{0}\}$ defined by $g\left(z_{1}, \ldots, z_{n}\right)=\left(\alpha_{1} z_{1}, \ldots, \alpha_{n} z_{n}\right)$, where $\alpha_{1}, \ldots, \alpha_{n}$ are constants with $\left|\alpha_{i}\right|>1(i=1, \ldots, n)$. Then $M\left(\alpha_{1}, \ldots, \alpha_{n}\right)$ is defined to be the quotient manifold $\mathbf{C}^{n} \backslash\{\mathbf{0}\} / G$, which is diffeomorphic to $S^{1} \times S^{2 n-1}$. Hence, its first Chern class vanishes (cf. [H]). There is a holomorphic fold map $f: M\left(\alpha_{1}, \ldots, \alpha_{n-1}, \alpha_{n}\right) \rightarrow M\left(\alpha_{1}, \ldots, \alpha_{n-1}, \alpha_{n}^{2}\right)$ defined by $f\left(\left[z_{1}, \ldots, z_{n-1}, z_{n}\right]\right)=\left[z_{1}, \ldots, z_{n-1}, z_{n}^{2}\right]$, where $[*]$ refers to the 
element represented by $*$. The singularity submanifold of $f$ is identified with $M\left(\alpha_{1}, \ldots, \alpha_{n-1}\right)$, which consists of the points of the form $\left[z_{1}, \ldots, z_{n-1}, 0\right]$.

(2) Given integers $a_{1}, \ldots, a_{n} \geq 2$, consider the Brieskorn polynomial $p(z)=z_{1}^{a_{1}}+\cdots+z_{n}^{a_{n}}(n \geq 2)$ and the hypersurface $p^{-1}(0)$. Let $r$ be a real number greater than 1 and $\alpha_{1}, \ldots, \alpha_{n}$ be $n$ complex numbers with $\alpha_{i}^{a_{i}}=r(i=1, \ldots, n)$. Then the group $G$ in $(1)$ acts on $p^{-1}(0) \backslash\{\mathbf{0}\}$. Let $B\left(a_{1}, \ldots, a_{n} ; \alpha_{1}, \ldots, \alpha_{n}\right)$ denote the quotient space $\left(p^{-1}(0) \backslash\{\mathbf{0}\}\right) / G$. Since $G$ is properly discontinuous (see $[\mathrm{K}$, Theorem 2.2]), it is a compact complex $n-1$ dimensional submanifold of $M\left(\alpha_{1}, \ldots, \alpha_{n}\right)$. Let $K\left(a_{1}, \ldots, a_{n}\right)$ be the Brieskorn manifolds $p^{-1}(0) \cap S_{\varepsilon}^{2 n-1}$, where $\varepsilon$ is a sufficiently small positive real number (see $[\mathrm{Br}]$ and $[\mathrm{Mi}])$. We can prove that $B\left(a_{1}, \ldots, a_{n} ; \alpha_{1}, \ldots, \alpha_{n}\right)$ is $C^{\infty}$-diffeomorphic to $S^{1} \times K\left(a_{1}, \ldots, a_{n}\right)$. We give a sketch of the proof, which is analogous to the arguments found in [K, Example 2.9].

For a real number $u$ and $\left(z_{1}, \ldots, z_{n}\right) \neq \mathbf{0}$, define the function $\mathcal{G}\left(u, z_{1}\right.$, $\left.\ldots, z_{n}\right)=\left|z_{1}\right|^{2}\left|\alpha_{1}\right|^{-2 u}+\cdots+\left|z_{n}\right|^{2}\left|\alpha_{n}\right|^{-2 u}$. Since $\lim _{u \rightarrow \infty} \mathcal{G}\left(u, z_{1}, \ldots, z_{n}\right)=$ $0, \lim _{u \rightarrow-\infty} \mathcal{G}\left(u, z_{1}, \ldots, z_{n}\right)=\infty$ and $\mathcal{G}\left(u, z_{1}, \ldots, z_{n}\right)$ is strictly decreasing with respect to $u$, the equation $\mathcal{G}\left(u, z_{1}, \ldots, z_{n}\right)=\varepsilon^{2}$ induces the unique implicit function $u(z)=u\left(z_{1}, \ldots, z_{n}\right)$. Consider the two $C^{\infty}$-maps,

$$
\begin{aligned}
& \Phi: \mathbf{R} \times K\left(a_{1}, \ldots, a_{n}\right) \longrightarrow p^{-1}(0) \backslash\{\mathbf{0}\} \\
& \Phi_{1}: p^{-1}(0) \backslash\{\mathbf{0}\} \longrightarrow \mathbf{R} \times K\left(a_{1}, \ldots, a_{n}\right)
\end{aligned}
$$

defined by $\Phi\left(u, \zeta_{1}, \ldots, \zeta_{n}\right)=\left(\alpha_{1}^{u} \zeta_{1}, \ldots, \alpha_{n}^{u} \zeta_{n}\right)$ and $\Phi_{1}\left(z_{1}, \ldots, z_{n}\right)=(u(z)$, $\left.\alpha_{1}^{-u(z)} z_{1}, \ldots, \alpha_{n}^{-u(z)} z_{n}\right)$ respectively. Since $\mathcal{G}\left(u, \alpha_{1}^{u} \zeta_{1}, \ldots, \alpha_{n}^{u} \zeta_{n}\right)=\left|\zeta_{1}\right|^{2}+$ $\cdots+\left|\zeta_{n}\right|^{2}=\varepsilon^{2}$, they satisfy that $\Phi_{1} \circ \Phi\left(u, \zeta_{1}, \ldots, \zeta_{n}\right)=\left(u, \zeta_{1}, \ldots, \zeta_{n}\right)$ and $\Phi \circ \Phi_{1}\left(z_{1}, \ldots, z_{n}\right)=\left(z_{1}, \ldots, z_{n}\right)$. Furthermore, we have the following commutative diagram:

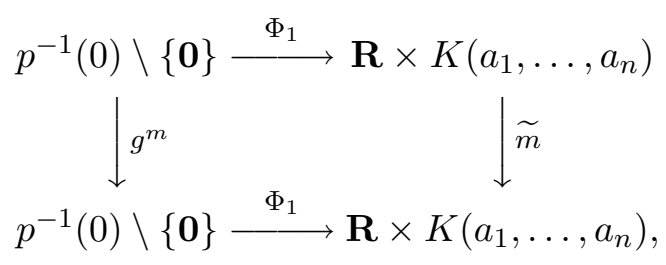

where $g^{m}\left(z_{1}, \ldots, z_{n}\right)=\left(\alpha_{1}^{m} z_{1}, \ldots, \alpha_{n}^{m} z_{n}\right)$ and $\widetilde{m}(u, \zeta)=(u+m, \zeta)$. This is what we want.

Note that the first Chern class of $B\left(a_{1}, \ldots, a_{n} ; \alpha_{1}, \ldots, \alpha_{n}\right)$ vanishes at least for $n \geq 4$ and $n=2$, since $K\left(a_{1}, \ldots, a_{n}\right)$ is simply connected 
for $n \geq 4$ ([Mi, Theorem 5.2]) and $\operatorname{dim} K\left(a_{1}, \ldots, a_{n}\right)=1$ for $n=2$. Furthermore $\operatorname{grad}(p(z))$ is equal to ${ }^{t}\left(a_{1} z_{1}^{a_{1}-1}, \ldots, a_{n} z_{n}^{a_{n}-1}\right)$, which cannot be orthogonal to all of the vectors $\mathbf{e}_{1}, \ldots, \mathbf{e}_{n-1}$. Hence, for any point $z$ of $p^{-1}(0) \backslash\{\mathbf{0}\}$, there exists a number $j$ with $1 \leq j \leq n-1$ such that $\left(z_{1}, \ldots, z_{j-1}, z_{j+1}, \ldots, z_{n}\right)$ is a local coordinate system both for $p^{-1}(0) \backslash\{\mathbf{0}\}$ near $z$ and for $B\left(a_{1}, \ldots, a_{n} ; \alpha_{1}, \ldots, \alpha_{n}\right)$ near $[z]$.

Let $\beta$ be a complex number with $\beta^{2}=\alpha_{n}$. Then we have the fold map $f: B\left(a_{1}, \ldots, a_{n-1}, 2 a_{n} ; \alpha_{1}, \ldots, \alpha_{n-1}, \beta\right) \rightarrow B\left(a_{1}, \ldots, a_{n-1}, a_{n} ; \alpha_{1}, \ldots, \alpha_{n-1}\right.$, $\left.\alpha_{n}\right)$ defined by $f\left(\left[z_{1}, \ldots, z_{n-1}, z_{n}\right]\right)=\left(\left[z_{1}, \ldots, z_{n-1}, z_{n}^{2}\right]\right)$. The singularity submanifold of $f$ is identified with $B\left(a_{1}, \ldots, a_{n-1} ; \alpha_{1}, \ldots, \alpha_{n-1}\right)$, which consists of the points of the form $\left[z_{1}, \ldots, z_{n-1}, 0\right]$ with $z_{1}^{a_{1}}+\cdots+z_{n-1}^{a_{n-1}}=0$.

In a forthcoming paper we will deal with a complex analogy of the results in $[$ An $2, \S 4]$. Let $F_{k}^{m}$ denote the space consisting of all continuous maps $\left(S^{k-1}, *\right) \rightarrow\left(S^{k-1}, *\right)$ of degree $m$, where $S^{k-1}$ is the unit sphere of dimension $k-1$ and $*$ is the base point. Let $F^{m}$ denote the space $\lim _{k \rightarrow \infty} F_{k}^{m}$. Let $N$ and $P$ be compact complex manifolds of dimension $n$ and $P$ be, in addition, connected. Then we will show that a holomorphic fold map $f: N \rightarrow P$ of degree $m$ determines a homotopy class of $\left[P, F^{m}\right]$, which depends only on a certain equivalence class of $f$.

\section{REFERENCES}

[A1] Y. Ando, The homotopy type of the space consisting of regular jets and folding jets in $J^{2}(n, n)$, Japan. J. Math., 24 (1998), 169-181.

[A2] Folding maps and the surgery theory on manifolds, J. Math. Soc. Japan, 53 (2001), 357-382.

[Bo] J. M. Boardman, Singularities of smooth mappings, Publ. Math. I.H.E.S., 33 (1967), 21-57.

[Br] E. Brieskorn, Beispiele zur Differentialtopologie von Singularitäten, Invent. Math., 2 (1966), 1-14.

[E] J. M. Eliashberg, On singularities of folding types, Math. USSR. Izv., 4 (1970), 1119-1134.

[H] F. Hirzebruch, Topological Methods in Algebraic Geometry, Springer-Verlag, 1966.

[K] K. Kodaira, Complex Manifolds and Deformation of Complex Structures, Springer-Verlag, 1986.

[L] H. I. Levine, Singularities of differentiable maps, Lecture Notes in Math., Springer-Verlag, 192 (1971), 1-89.

[Ma] J. Mather, Stability of $C^{\infty}$ mappings: VI. The nice dimensions, Lecture Notes in Math., Springer-Verlag, 192 (1971), 207-253. 
[Mi] J. Milnor, Singular Points of Complex Hypersurfaces, Princeton Univ. Press, Princeton, 1968.

[Sa] O. Saeki, Notes on the topology of folds, J. Math. Soc. Japan, 44 (1992), 551-566.

[St] N. Steenrod, The Topology of Fibre Bundles, Princeton Univ. Press, Princeton, 1951.

[W] H. Whitney, Complex Analytic Varieties, Addison-Wesley, 1972.

Department of Mathematics

Faculty of Science

Yamaguchi University

Yamaguchi, 753-8512

Japan

andoy@po.cc.yamaguchi-u.ac.jp 